\title{
Vom situationalen Interesse zum Lernerfolg: Lesegeschichten im Chemieunterricht der Sekundarstufe I
}

\author{
Tim Reschke $^{1}$ (D) Jenna Koenen ${ }^{2} \cdot$ Elke Sumfleth $^{1}$ \\ Eingegangen: 6. Februar 2020 / Angenommen: 3. Oktober 2020 / Online publiziert: 3. November 2020 \\ (c) Der/die Autor(en) 2020
}

\section{Zusammenfassung}

Schülerinnen und Schüler zeigen im Fach Chemie häufig Defizite im Fachwissen und gleichzeitig ein geringes Fachinteresse. Eine vermehrte Anregung des situationalen Interesses könnte einen ersten Schritt in Richtung einer positiven Entwicklung des Fachinteresses sein. Eine Möglichkeit hierzu bieten Lernmaterialien in Form von chemiebezogenen Lesegeschichten. Allerdings mangelt es an empirischen Studien zur Wirkung solcher Texte auf das situationale Interesse und den Lernerfolg. Darüber hinaus fehlt ein Vergleich mit anderen Textarten wie dem Sachtext, der meist vorkommenden Textart in Chemieschulbüchern. Im Rahmen der vorliegenden Studie wurden daher verschiedene Lesegeschichten entwickelt und untersucht, inwieweit sich diese bei fachlich gleichen Inhalten in ihrer Wirkung von Sachtexten unterscheiden, und zwar im Hinblick auf das situationale Interesse und den Lernerfolg von Schülerinnen und Schülern. Die Ergebnisse zeigen, dass der Lernerfolg mit Lesegeschichten höher ist als der mit Sachtexten. Beim situationalen Interesse zeigen sich Geschlechtereffekte zugunsten der Mädchen, die stärker an der Lesegeschichte interessiert sind und dadurch länger mit dieser lernen als die Jungen. Insgesamt lässt sich für die Mädchen ein Modell hinsichtlich des Zusammenhangs zwischen der jeweiligen Textart (mit Vorteilen für die Lesegeschichte), dem situationalen Interesse, der Lernzeit und dem Lernerfolg abbilden.

Schlüsselwörter Lesegeschichten · Situationales Interesse · Lernerfolg · Geschlechtereffekte

\section{Situational Interest and Its Influence on Students' Content Knowledge: The Use of Narrative Texts in Chemistry Lessons}

\begin{abstract}
Students show a lack in content knowledge and are often less interested in Chemistry. As it is well known that a permanent stimulation of situational interest can positively influence students' individual interest, learning materials (e.g. in form of narrative texts) may foster students' situational interest and increase students' knowledge. However, there is a lack of empirical studies concerning narrative texts in comparison to other text types like expository texts, which is the dominant form of schoolbook texts. Therefore, the purpose of this study is the development and evaluation of a narrative text to investigate the influence on the enhancement of students' domain-specific content knowledge and on the increase of their situational interest comparing to expository texts including the same learning content. Results of the study show that the group, which is learning with the narrative text, achieves a significant higher knowledge growth and has a higher situational interest than the group learning with the expository text. In addition, there are gender effects with regard to studying with the narrative text. Girls are more interested in the narrative text, rate the text more positively and spend more time with the text than boys. All in all, there is a valid model for girls showing a relation between the narrative text, situational interest, learning time and learning achievement.
\end{abstract}

Tim Reschke

tim.reschke@web.de

1 Didaktik der Chemie, Universität Duisburg-Essen, Schützenbahn 70, 45127 Essen, Deutschland

2 Didaktik der Chemie, Technische Universität München, Arcisstraße 21, 80333 München, Deutschland 


\section{Einführung}

Der deutschlandweite Bildungstrend von 2018 vom Institut zur Qualitätsentwicklung im Bildungswesen (IQB) zeigt, dass Schülerinnen und Schüler in der Sekundarstufe I im Fach Chemie häufig Defizite im Bereich des Fachwissens haben (vgl. Stanat et al. 2018). Des Weiteren macht der Ländervergleich deutlich, dass die Schülerinnen und Schüler ein geringes Interesse am Fach haben. Eine vermehrte Anregung des situationalen Interesses könnte einen ersten Schritt in Richtung einer positiven Entwicklung des Fachinteresses darstellen (vgl. Hidi und Berndorff 1998) und darüber hinaus Einfluss auf den Lernerfolg im Bereich des Fachwissens nehmen (vgl. sowie Abschn. Vom Interesse zum Lernerfolg).

Höft et al. (2019) konnten zeigen, dass das Interesse von Schülerinnen und Schülern an naturwissenschaftlichen Schulaktivitäten mehr oder weniger monoton im Verlaufe der Sekundarstufe I abnimmt. Darüber hinaus zeigt sich, dass vor allen Dingen in höheren Jahrgangstufen ein $\mathrm{Zu}$ sammenhang zwischen dem Interesse an Aktivitäten, die kognitiv aktivierend sind sowie eine kommunikative Komponente besitzen, und dem Lernerfolg besteht (Höft et al. 2019). Eine Adressierung des Interesses an solchen Aktivitäten durch den Einsatz spezifischer Unterrichtsmethoden oder Materialien könnte eine Möglichkeit darstellen, um den Lernerfolg zu steigern.

Eine Möglichkeit könnten hierbei Lerngelegenheiten in Form von chemiebezogenen Lesegeschichten bieten (vgl. Avraamidou und Osborne 2009; vgl. Kap. 2.2.2). Erste Befunde konnten zeigen, dass sich anhand einer Lesegeschichte konkrete Fachinhalte im Fach Physik vermitteln lassen (Kaspar und Mikelskis 2008). Allerdings postulieren Avraamidou und Osborne (2009), dass es kaum empirische Studien zum Einsatz von Lesegeschichten im naturwissenschaftlichen Unterricht gibt, insbesondere nicht zur Wirkung auf das situationale Interesse und die Lernförderlichkeit. Darüber hinaus gibt es kaum Vergleichsstudien mit anderen Textsorten wie zum Beispiel Sachtexten (Wellington und Osborne 2001). Ein Blick in verschiedene Chemieschulbücher zeigt, dass sich ein solcher Vergleich jedoch anbietet, da diese Textsorte am häufigsten vorkommt. Die Problematik, die bei fast allen Sachtexten aufkommt, ist, dass diese Texte oft zu viele Informationen und zu viele unbekannte Fachwörter in einem kurzen Abschnitt enthalten, sodass die Fachsprache von den Schülerinnen und Schülern häufig als zu schwierig empfunden wird (vgl. Beerenwinkel und Gräsel 2005). Jedoch ist noch weitestgehend ungeklärt, inwieweit solche Merkmale auf lexikalisch-syntaktischer Ebene tatsächlich zu Verstehensschwierigkeiten führen (vgl. Heine et al. 2018). Unstrittig ist jedoch, dass Sachtexte im Vergleich zu Lesegeschichten andere narrative Merkmale enthalten (vgl. Avraamidou und Osborne 2009). In dieser
Studie werden daher Lesegeschichten entwickelt und mit Sachtexten zu identischen Inhalten verglichen. Hierbei soll untersucht werden, inwieweit sich das Lernen mit Lesegeschichten im Vergleich zum Lernen mit Sachtexten auf das situationale Interesse und den Lernerfolg der Schülerinnen und Schüler auswirkt.

\section{Theoretischer Hintergrund}

\section{Vom Interesse zum Lernerfolg}

Aus Sicht der pädagogischen Psychologie stellt das Interesse für das schulische Lernen eine wichtige Komponente dar. Krapp $(1992,2002)$ unterscheidet zwischen dem individuellen und dem situationalen Interesse. Unter individuellem Interesse wird eine ,persönlichkeitsspezifische Vorliebe für ein bestimmtes Wissens- oder Handlungsgebiet" wie beispielsweise das Interesse an einem Fachgebiet verstanden (Höft et al. 2019; Krapp 1992; Rotgans und Schmidt 2017). Dieses entwickelt sich über eine lange Zeit (Hidi 2006; Krapp 1992, 2002) und gilt als relativ stabiles Personenmerkmal (Schmidt und Rotgans 2017). Demgegenüber ist das situationale Interesse situationsspezifisch und lässt sich durch Merkmale der Situation beeinflussen (Höft et al. 2019; Krapp 1992, 2002).

Beide Konstrukte lassen sich theoretisch und empirisch voneinander trennen, wechselwirken jedoch miteinander. Krapp (1992) geht davon aus, dass das situationale Interesse durch die Interaktion zwischen dem individuellen Interesse einer Person und der Interessantheit eines Gegenstands entsteht. Rotgans und Schmidt (2014) gehen hingegen nehmen an, dass situationales Interesse entsteht, wenn einer Person bewusstwird, dass sie Wissenslücken in einem bestimmten Bereich aufweist. Dies resultiert in einer vertieften Beschäftigung mit der Thematik und führt somit zum Lernen.

Nach Hidi und Berndorff (1998) kann durch Aufrechterhalten des situationalen Interesses über einen längeren Zeitraum das individuelle Interesse positiv beeinflusst werden. Rotgans und Schmidt (2017) postulieren, dass diese beiden Interessensarten im Wesentlichen durch Lernen verbunden werden. So wird angenommen, dass die Erzeugung von situationalem Interesse zu Lernen führt und individuelles Interesse wiederum ein Ergebnis dieses Lernprozesses ist. Demnach kann das situationale Interesse einen geeigneten Ausgangspunkt für Lernerfolg darstellen. Des Weiteren wird angenommen, dass situationales Interesse durch instruktionelle Stimuli erzeugt werden kann (u.a. Tapola et al. 2013).

Dazu zählt beispielsweise auch die Interessantheit eines Textes (text-based interest) (Hidi und Baird 1988). Hidi und Baird (1988) konnten zeigen, dass die Interessantheit eines Textes einen positiven Einfluss auf das situationale Inte- 
resse hat. Schraw und Lehman (2001) arbeiten in diesem Zusammenhang empirisch drei Faktoren heraus, die das textbezogene Interesse beeinflussen: seductiveness, vividness und coherence. Seductiveness basiert auf dem Einsatz von interessanten Details (seductive details), die die Leserin und den Leser für gewisse Textpassagen ,,packen“ sollen; diese können jedoch auch ablenkend wirken (Hidi und Baird 1988). Vividness bezieht sich auf die Lebendigkeit eines Textes, die beispielsweise durch spannende und überraschende Textpassagen erreicht werden kann (z. B. Garner 1992). Bei der coherence handelt es sich um einen Strukturparameter wie z. B. einen roten Faden, der es der Leserin und dem Leser erleichtert, die Hauptgedanken aus dem Text nachzuvollziehen (z. B. Campbell 1995). Darüber hinaus konnten verschiedene Studien zeigen, dass sich das situationale Interesse positiv auf das Lernen auswirkt (z. B. Hulleman et al. 2010) Daher stehen im schulischen Kontext Lernumgebungen als Interessensgegenstand im Fokus. Es kann gezielt Einfluss auf die Gestaltung eines Lernmaterials genommen und so die Interessantheit des Gegenstands beeinflusst werden (Krapp 1992, 2002). Eine Möglichkeit hierfür können Lerngelegenheiten in Form von Geschichten bieten (Avraamidou und Osborne 2009).

\section{Wirkung von Geschichten auf affektive Variablen und Lernerfolg}

Egan (1988) stellt fest, dass sich die Schülerinnen und Schüler zunächst affektiv von der Geschichte angesprochen fühlen müssen, um sich dann schließlich kognitiv mit den neuen Fachinhalten auseinanderzusetzen. Viele andere Arbeiten halten die Verknüpfung der affektiven und kognitiven Komponenten durch den Einsatz von Geschichten für genauso wichtig (z.B. Abrahamson 1998; Collins 1999; Giessen 2009; Heering 2013). Affektive Komponenten beim Umgang mit einer Geschichte können Motivation (z. B. Lück 2006; Kubli 2002, 2005), das konkrete Interesse an der Geschichte (situationales Interesse) (z. B. Martensen et al. 2007) sowie Freude (z. B. Andrews et al. 2009; Folino 2001) sein. Lernpsychologinnen und Lernpsychologen stellen in diesem Zusammenhang immer wieder heraus, dass das menschliche Gedächtnis für Geschichten gut zugänglich ist, weil anhand von Geschichten die Verknüpfung der affektiven und kognitiven Komponenten angesprochen wird (z. B. Berliner 1992). Wenn man sich von einer Geschichte angesprochen fühlt, dann kann man sich gut an die Handlung der Geschichte zurückerinnern (Martin 1999). In diesem Zusammenhang untersuchten Kaspar und Mikelskis (2008) in der Sekundarstufe I narrativ gestaltete Dialoge mit einer konkreten Handlung hinsichtlich ihrer Wirkung auf den Lernerfolg. Ergebnisse ihrer Untersuchung zeigen, dass die Schülerinnen und Schüler die narrative Lernumgebung sowie die dargestellten Figuren für ansprechend hiel- ten. Die Schülerinnen und Schüler erreichten signifikante Fachwissenszuwächse, die auch nach mehreren Wochen noch vorhanden waren. Martensen et al. (2007) untersuchten im Chemieunterricht ebenfalls in der Sekundarstufe I die Wirkung von mehreren Geschichten in einer Lernumgebung zum Thema „Strom durch Chemie“ auf das situationale Interesse und den Lernerfolg. Die Ergebnisse dieser Studie zeigen, dass sich die Schülerinnen und Schüler mit den Geschichten Fachwissen aneignen konnten. Darüber hinaus waren sie an den Geschichten interessiert. Mädchen waren insgesamt stärker an den Geschichten interessiert als Jungen (Martensen et al. 2007) und arbeiteten lieber mit den Geschichten. Die Frage, ob sich die Schülerinnen und Schüler vorstellen können, erneut mit Geschichten chemiespezifische Inhalte zu lernen, bejahten $75 \%$ aller Mädchen, aber nur die Hälfte der Jungen. In der Studie kam außerdem zum Ausdruck, dass Schülerinnen und Schüler mit geringem Fachinteresse besonders gern mit den Geschichten lernten. Das galt auch für Schülerinnen und Schüler, die das Fach Chemie vor der Intervention als schwierig einschätzten. In einer Studie von McQuiggan et al. (2008) wurden geschichtenbasierte Lernumgebungen anhand einer virtuellen Welt zum Thema Mikrobiologie eingesetzt, um deren Einfluss auf den Lernerfolg, die Aufmerksamkeit sowie das situationale Interesse der Schülerinnen und Schüler $\mathrm{zu}$ untersuchen. Hierbei wurden eine geschichtenbasierte Lernumgebung (narrative condition) mit einer teilweise geschichtenbasierten Lernumgebung (minimal-narrative condition) sowie mit einer Lernumgebung ohne Geschichten verglichen. In der narrative condition erhielten die Schülerinnen und Schüler eine Storyline mit mehreren Charakteren. Im Unterschied zu der narrative condition bekamen die Schülerinnen und Schüler in der minimal-narrative condition eine gekürzte Storyline ohne Charaktere. Ergebnisse dieser Studie zeigen, dass Schülerinnen und Schüler in der narrative condition die höchste Aufmerksamkeit hatten. Auch hier zeigte sich ein Geschlechtereffekt. Mädchen hatten im Vergleich zu Jungen in beiden narrativen Lernumgebungen eine signifikant höhere Aufmerksamkeit beim Lernen. Darüber hinaus korrelierte das situationale Interesse signifikant mit der Aufmerksamkeit ( $r=0,36$ mit $p<0,01)$.

Je höher das situationale Interesse war, desto höher war die Aufmerksamkeit. Die Schülerinnen und Schüler zeigten in der narrative condition signifikant mehr Aufmerksamkeit als die Schülerinnen und Schüler in der minimal condition. Hinsichtlich des Lernerfolgs zeigen die Ergebnisse, dass die Schülerinnen und Schüler in allen Gruppen einen signifikanten Lernzuwachs vom Prä- zum Post-Messzeitpunkt erreichten. Einen Zusammenhang zwischen der Aufmerksamkeit und des Lernerfolgs haben McQuiggan et al. (2008) jedoch nicht feststellen können. Schraw (1997) konnte zuvor die Wirkung verschiedener Strukturmerkmale eines narrativ-literarischen Textes auf das situationale Inte- 
resse identifizieren. Dafür entwickelte er einen „Sources of Interest Questionnaire" (SIQ) und konnte zeigen, dass die Kohärenz (coherence), die thematische Komplexität (thematic complexity) und die Spannung des Textes (suspense) Prädiktoren für das situationale Interesse sind. Jedoch bleibt insgesamt festzuhalten, dass keine der vorherig genannten Studien bisher einen Zusammenhang vom situationalen Interesse und dem Lernerfolg durch das Lernen mit einer Lesegeschichte zeigen konnte.

\section{Narrative Textelemente in Geschichten}

Allgemein liegen einer Lesegeschichte wichtige narrative Elemente zugrunde (Fludernik 2006). Hierzu geben Avraamidou und Osborne (2009) eine Übersicht über grundlegende Elemente narrativer Texte. Eine Geschichte ist zunächst eine Aneinanderreihung von Geschehnissen (chain of events), die einen sogenannten Plot, beinhaltet. Der Plot sollte hierbei chronologisch geordnet sein (Norris et al. 2005), die Struktur durch einen Spannungsaufbau, beginnend mit einem Konflikt und endend mit einer Lösung, gekennzeichnet sein (structure) und bereits Geschehenes wiedergeben (time) werden (vgl. Avraamidou und Osborne 2009). Die Geschichte muss von einer erzählenden Person wiedergegeben werden. Die erzählende Person kann sich im Hintergrund der Geschichte befinden oder eine aktive Person in der Geschichte sein (narrator) (vgl. Avraamidou und Osborne 2009). Hierbei kann die Perspektive der erzählenden Person, auch als Fokalisierung oder Point of View bezeichnet, entscheidend sein (Genette 2010). Ist eine erzählende Person direkt an der Geschichte beteiligt und stellt sogar eine Hauptfigur dar, so ist der höchste Grad der Fokalisierung erreicht. Bei einer solchen erzählenden Person handelt es sich oft um ein erzählendes Ich (Stanzel 1998). Jahrhaus (2008) betont in diesem Zusammenhang, dass immer ein Wechselspiel zwischen einer Ich- und einer Du-Erzählung vorhanden ist, da ein „Du“ auch immer von einem „Ich“ und umgekehrt geäußert wird. Bei einer Du-Person ist das Merkmal der „Adressivität“, welches nach Kubli (2001) ein wichtiges Qualitätsmerkmal von Geschichten ist, vorhanden. Eine Du-Person als gezielten Adressaten kann den Identifikationsgrad erhöhen. Es gibt Studien, die belegen, dass Schülerinnen und Schüler dann besser lernen, wenn sie in einem Text direkt angesprochen werden (z. B. Mayer 2009). Diese Art der Ansprache wird als Personalisierungsprinzip (personalization principle) bezeichnet und zeichnet sich durch ein ,gesprächsartigeres" Sprachregister aus, wodurch im Gegensatz zum formalen Sprachregister ein besserer Bezug zur Alltagssprache erreicht werden kann (Mayer 2009). Studien konnten in diesem Zusammenhang zeigen, dass Mädchen eher weibliche und Jungen eher männliche Hauptfiguren bevorzugen (z.B. Ainley et al. 2002). Die Identifikation mit einer Geschichte kann durch einen so- genannten narrative appetite verstärkt werden (vgl. Norris et al. 2005). Dies bedeutet, dass die Geschichte so gestaltet sein sollte, dass die Leserin und der Leser unbedingt wissen will, wie es in der Handlung weitergeht. Die Handlung kann durch die Wahl geeigneter Figuren unterstützt werden. Hierbei muss es sich nicht zwingend um menschliche Figuren handeln, sondern auch things (vgl. Chatman 1978), quasi-humans (vgl. Toolan 2001) sowie material entities (vgl. Avraamidou und Osborne 2009) können als handelnde Figuren in der Geschichte fungieren, welches als Personifikation bezeichnet wird. Im naturwissenschaftlichen Unterricht ist diese auch unter der Bezeichnung „Anthropomorphismus“ (z.B. Davies 2010; Talanquer 2013) oder „Animismus“ (vgl. Püttschneider und Lück 2004) bekannt. Der Einsatz dient in erster Linie dazu, durch Rückgriff auf bereits bekannte und vertraute Sachverhalte aus dem Alltag der Schülerinnen und Schüler Anknüpfungspunkte für neue Inhalte zu schaffen, wodurch der Unterricht für die Schülerinnen und Schüler auch motivierender gestaltet werden kann (Miller 1992; Püttschneider und Lück 2004). Allerdings können solche Anthropomorphismen auch unangemessene Vorstellungen generieren (Kattmann 2005). Püttschneider und Lück (2004) setzten animistische Bilder im Chemieunterricht der Jahrgangsstufe 7 und 8 ein und konnten zeigen, dass Schülerinnen und Schüler die dargestellten Animismen nicht als Abbild der Realität auffassten und ihnen durchaus bewusst war, dass Atome nicht sprechen können. Darüber hinaus kann eine bewusste Wahl der Sprache das Verständnis der Schülerinnen und Schüler fördern. Stilmittel wie Analogien, Metaphern und Übertreibungen können beispielsweise unterstützend für das Verständnis der fachlichen Textinhalte sein (z.B. Duit und Glynn 1992; Glynn 2007). Zusätzlich kann der Neologismus als ein weiteres Stilmittel für die Namensgestaltung von Figuren verwendet werden (Autor 2013). Bei diesem Stilmittel handelt es sich um eine Wortneuschöpfung (Göttert und Jungen 2004), die durch ein morphologisches Verfahren beispielsweise in Form der Suffigierung (Fleischer und Barz 2012) gebildet werden kann. Bei diesem Prozess werden an Wortstämme Suffixe gehängt. Als Suffixe können beispielsweise sogenannte produktive Diminutivsuffixe - verniedlichende Suffixe - dienen (Dressler und Barbaresi 1994). Zusätzlich können die Dimensionen der Textverständlichkeit von Langer et al. (2011) bei der Gestaltung von Texten helfen. Diese haben die vier Dimensionen Einfachheit, Gliederung/ Ordnung, Kürze/Prägnanz und zusätzliche Stimulanz zur Verständlichkeit eines Textes identifiziert. Hierbei bezieht sich Einfachheit auf die sprachliche Gestaltung des Textes. Diese kann durch einfache Satzstrukturen und geläufige Wörter aus der Alltagssprache erreicht werden. Aktive Formulierungen und der Verzicht auf Substantivierungen können ebenfalls dazu beitragen. Die Dimension Gliederung/ Ordnung beschreibt die Struktur eines Textes. Absätze kön- 
nen dabei die Struktur unterstützen. Kürze/Prägnanz zielt auf das Vermeiden redundanter oder irrelevanter Informationen ab. Die zusätzliche Stimulanz kann bei der Gestaltung von Texten einen besonderen Stellenwert einnehmen. Diese kann beispielsweise durch wörtliche Reden, Verwendung von rhetorischen Mitteln, Auftreten von Figuren etc. umgesetzt werden. Der Gebrauch dieser Merkmale zur zusätzlichen Stimulanz sollte allerdings gut portioniert eingesetzt werden, damit die Übersichtlichkeit der Inhalte sowie das Gleichgewicht zwischen Kürze und Redundanz bestehen bleiben. Es gilt, die in diesem Kapitel dargestellten Merkmale bei der Materialentwicklung zu berücksichtigen.

\section{Studie}

\section{Forschungsfragen und Design}

Die Ziele der Studie sind die Unterstützung des situationalen Interesses und die Förderung des Lernens im Bereich des Fachwissens durch Lesegeschichten in der Jahrgangsstufe 8 an Gymnasien. Lesegeschichten werden mit Sachtexten, im Hinblick auf das situationale Interesse und das Lernen verglichen. Sachtexte werden oft als schwierig empfunden, da sie in einem kurzen Abschnitt viele Fachinhalte mit unbekannten Fachwörtern enthalten (vgl. Beerenwinkel und Gräsel 2005). Anhand beider Textsorten wird neues Fachwissen zu den Alkalimetallen vermittelt. Die entwickelten Lernmaterialien wurden zuvor in einer Interviewstudie erprobt (vgl. Autor 2017), um mögliche Schwierigkeiten beim Lernen zu identifizieren. In dieser Interventionsstudie wurde pro Textsorte nur ein Beispiel verwendet, da in einer Vorstudie anhand des Einsatzes von zwei Lesegeschichten zu unterschiedlichen Themen gezeigt werden konnte, dass kein Neuigkeitseffekt durch den erstmaligen Einsatz solcher Lesegeschichten auftrat (vgl. Autor 2017). Insgesamt ergeben sich daher für die Studie die folgenden Forschungsfragen:

1. Inwieweit können Schülerinnen und Schüler mit der Lesegeschichte besser unbekannte Fachinhalte erlernen als mit dem Sachtext?

2. Inwieweit unterscheidet sich das situationale Interesse beim Lernen zwischen der Lesegeschichte und dem Sachtext?
3. Inwieweit unterscheidet sich die Lernzeit zwischen Lesegeschichte und Sachtext?

4. Inwieweit treten Geschlechtereffekte beim Lernen mit der Lesegeschichte und dem Sachtext hinsichtlich des Lernerfolgs, des situationalen Interesses und der Lernzeit auf?

Des Weiteren soll untersucht werden, inwiefern Kontrollvariablen wie zum Beispiel die aktuelle Motivation, kognitive Fähigkeiten sowie Prozessvariablen wie der Cognitive Load und die Lernzeit Einfluss auf den Lernerfolg haben, sodass die vierte Forschungsfrage lautet:

5. Inwieweit nehmen Kontrollvariablen wie die aktuelle Motivation, kognitive Fähigkeiten sowie Prozessvariablen wie der Cognitive Load und Einfluss auf den Lernerfolg?

Damit ergeben sich für das Untersuchungsdesign zwei Interventionsgruppen: eine Geschichts- und eine SachtextGruppe. Die Geschichte und der Sachtext werden in den Klassen randomisiert verteilt. An einem ersten Tag in einer Schulstunde von 45 min werden Prä-Testinstrumente eingesetzt. Eine Woche später in einer weiteren Schulstunde findet die Intervention mit den beiden Gruppen und begleitenden Testinstrumenten sowie der Post-Tests statt (insgesamt $60 \mathrm{~min}$ ). Vier bis sechs Wochen später werden in 30 min Follow-up-Testinstrumente eingesetzt. Der Ablauf der Interventionsstudie sieht wie folgt aus (Abb. 1):

\section{Entwicklung der Lernmaterialien}

Die Themen zu den Texten stammen aus den Inhaltsfeldern des Kernlehrplans des Ministeriums für Schule und Weiterbildung des Landes Nordrhein-Westfalen für die Sekundarstufe I an Gymnasien (vgl. Kernlernplan NRW für Gymnasien 2008). Es wird das Thema ,Alkalimetalle“, das aus dem übergeordneten Inhaltsfeld „Elementfamilien, Atombau und Periodensystem“ stammt, betrachtet. Da die Alkalimetalle eine Elementfamilie des Periodensystems bilden, lässt sich das Thema der Stufe II (,Zusammenhänge erkennen“) in dem Basiskonzept „Struktur der Materie“ zuordnen. Hierzu gehört, dass „, die Schülerinnen und Schüler das Konzept zur Struktur der Materie soweit differenziert haben, dass sie Aufbauprinzipien des Periodensystems der Elemente beschreiben und als Ordnungs- und Klassifikationssche-
Abb. 1 Ablauf der Interventionsstudie nach Reschke (2017)

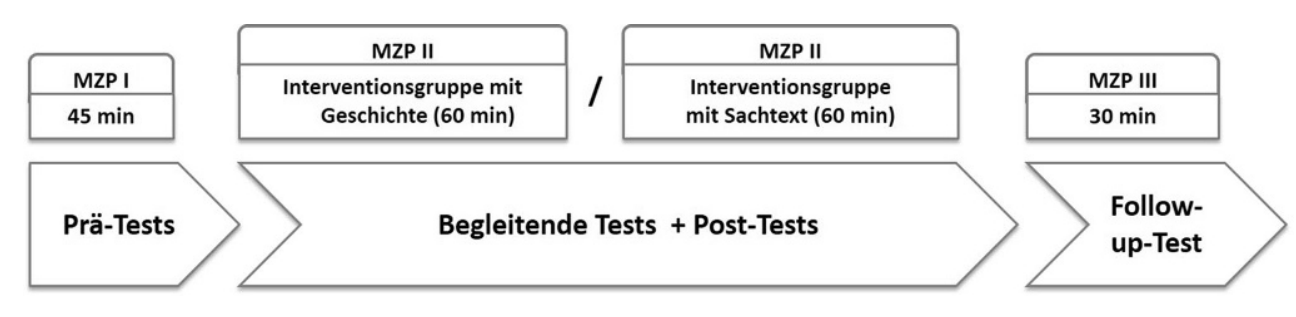


Tab. 1 Unterschiede hinsichtlich der strukturellen Merkmale bei der Entwicklung der Texte nach Reschke (2017)

\begin{tabular}{lll}
\hline Merkmale & Lesegeschichte & Sachtext \\
\hline $\begin{array}{l}\text { Figuren } \\
\text { Erzählweise }\end{array}$ & Vorhanden & - \\
Ich-Du-Erzählung & $\begin{array}{l}\text { Man- und Passiv- } \\
\text { konstruktionen }\end{array}$ \\
Wiedergabe & $\begin{array}{l}\text { Vergangenheitstempora } \\
\text { und Präsens }\end{array}$ & Präsens \\
Sprache & Alltags- und Fachsprache & Fachsprache \\
Rhetorische & Personifikation, Analogi- & - \\
Mittel & en, Neologismus & \\
Zusätzliches & Diminutivsuffix & - \\
\hline
\end{tabular}

ma nutzen und Haupt- und Nebengruppen unterscheiden " (Kernlehrplan NRW 2008, S. 28). Aufgrund des Gefahrenpotentials von Alkalimetallen dürfen Versuche mit Lithium und Natrium nur lehrerzentriert und unter bestimmten Sicherheitsvorkehrungen durchgeführt werden. Ein Einsatz von Versuchen mit Kalium und den folgenden Alkalimetallen ist sogar untersagt. Daher bieten Texte eine mögliche Alternative, um Gefahren durch einen Versuch mit einem Alkalimetall gänzlich auszuschließen. Des Weiteren bietet der Einsatz von Texten eine schülerzentrierte Perspektive, sodass die Schülerinnen und Schüler aktiv am Lernprozess teilhaben können.

Bei der Entwicklung beider Textsorten (siehe Links zum Download) sind aufgrund der theoretischen Grundlagen verschiedene strukturelle Merkmale umgesetzt worden (vgl. Tab. 1).

In den Lesegeschichten werden Figuren verwendet. Als Erzählweise wird in den Lesegeschichten eine Ich-Du-Erzählung verwendet. Das Ziel ist hierbei, dass sich die Leserinnen und Leser in die erzählte Geschichte hineinversetzen und mit dem „Du“ identifizieren können. Das „Du“ ist so dargestellt, dass ungeklärt bleibt, um welche Du-Person es sich handelt (vgl. Abschn. Narrative Textelemente in Geschichten). Die Leserinnen und Leser wissen nur, dass die Person eine nahestehende Person ist - beispielsweise eine Partnerin, ein Partner, eine gute Freundin, ein Freund etc. Das Geschlecht der Du-Person bleibt zudem völlig offen. Das erzählende Ich ist zugleich Hauptfigur der Geschichte. Dieser schildert dabei nicht nur die Handlung, sondern auch Gefühle aller beteiligten Hauptfiguren, sodass der höchste Grad der Fokalisierung vorhanden ist (vgl. Abschn. Narrative Textelemente in Geschichten). Die erzählende Person verwendet bei der Darstellung der Fachinhalte Fachsprache, allerdings gibt er die wörtliche Rede der Anthropomorphismen in der Alltagssprache wieder. In dem folgenden Beispiel gibt die erzählende Person das Gesagte des Elementes Kalium wieder: „Oh cool, Natri, bei dir zischt es ja noch mehr und du bewegst dich noch schneller hin und her! Du gehst eindeutig mehr ab als Lithi [...]“. Die Verwendung rhetorischer Mittel unterstützt die Alltagssprache. Das
Merkmal der Personifikation ist in diesem Zusammenhang besonders ausgeprägt. Es zeigt sich in den Lesegeschichten durch die Vermenschlichung von Elementen, sodass Anthropomorphismen entstehen (vgl. Abschn. Narrative Textelemente in Geschichten). Diese nutzen in ihrer Sprache Analogien aus bekannten und vertrauten Inhalten des Alltags, um die unbekannten Fachinhalte zu veranschaulichen. Darüber wurden die Namen im Sinne des Neologismus durch das verniedlichende Diminutivsuffix -i gebildet (vgl. Abschn. Narrative Textelemente in Geschichten). So wurde in den Elementnamen das Suffix - um durch das Suffix - $i$ ersetzt. In einer Studie ( Reschke 2013) konnte gezeigt werden, dass die Namen von den Schülerinnen und Schülern positiv bewertet wurden. Der Sachtext ist hingegen in einem formalen Stil anhand von man- und Passivkonstruktionen im Präsens gestaltet und enthält ausschließlich Fachsprache (vgl. Reschke 2017). Bei der Entwicklung beider Texte wurden die Dimensionen des Hamburger Textverständlichkeitsmodells berücksichtigt (vgl. Abschn. Narrative Textelemente in Geschichten) - mit der Ausnahme, dass beim Sachtext die zusätzliche Stimulanz aufgrund der Unterscheidung zur Lesegeschichte nicht angewandt wurde. Nach der Entwicklung wurden beide Texte anhand des Flesch-Indexes (Flesch 1951) auf ihre Lesbarkeit geprüft. Für die Lesegeschichte werden gute Lesbarkeitswerte erreicht. Die Werte betragen 64 von 100 (leichterlesbar.ch) und 65 von 100 (fleschindex.de). Damit liegt die Lesegeschichte hinsichtlich der Lesbarkeit für die 8. Jahrgangsstufe des Gymnasiums in einem angemessenen Bereich. Da der Sachtext an Lehrtexte zum gleichen Thema aus Chemieschulbüchern (z. B. Asselborn et al. 2009) angelehnt ist, um einen QuasiVergleich zwischen solchen Sachtexten und Lesegeschichten durchzuführen, liegt der Lesbarkeitswert des Sachtextes im Bereich der Lesbarkeit gängiger Schulbüchern. Für den Sachtext zum Thema „Alkalimetalle“ liegen Werte von 46 (leichterlesbar.ch) und 45 (fleschindex.de) vor. Der Sachtext befindet sich damit in einem durchschnittlichen Lesbarkeitsbereich. Damit liegen die Werte immer noch leicht über den Lesbarkeitswerten von Chemieschulbüchern (Werte von 20 bis 40), sodass der entwickelte Sachtext in dieser Arbeit geringfügig leichter lesbar ist. Dies wurde bewusst so gewählt, um sich der Kritik von Lehrkräften, Lehrtexte in den Chemieschulbüchern seien im Hinblick auf Sprache und Verständlichkeit zu schwierig, zu stellen (vgl. Beerenwinkel und Gräsel 2005). Die Lesegeschichte ist zwar deutlich länger als der Sachtext, jedoch sind diese fachinhaltlich identisch. Den Schülerinnen und Schüler war es während der Lernphase jederzeit gestattet, ihren Lernprozess zu beenden. Sie konnten daher selber entscheiden, wie lange und ob sie den jeweiligen Text lesen. Der Arbeitsauftrag lautete dabei, dass sie den Text lesen sollen und dass sie dabei alles tun dürften, was sie sonst auch tun, wenn sie mithilfe eines Textes lernen. 
Tab. 2 Eingesetzten Fragebögen und Testinstrumente (nach Reschke 2017)

\begin{tabular}{lllll}
\hline Testinstrumente/Fragebögen & \multicolumn{3}{l}{ Messzeitpunkte } \\
\cline { 2 - 5 } & Prä & Begleitend & Post & Follow up \\
\hline Fachwissenstest & $\mathrm{x}$ & - & $\mathrm{x}$ & $\mathrm{x}$ \\
Fragebogen zur Lesemotivation & $\mathrm{x}$ & - & - & - \\
Kognitiver-Fähigkeiten-Test N2 & $\mathrm{x}$ & - & - & - \\
LGVT (Leseverständnis) & - & - & - & $\mathrm{x}$ \\
FAM (aktuelle Motivation) & - & $\mathrm{x}$ & - & - \\
Lernzeit & - & $\mathrm{x}$ & - & - \\
Cognitive Load & - & $\mathrm{x}$ & - & - \\
Fragebogen zum situationalen Interesse nach Text & - & $\mathrm{x}$ & - & - \\
und Thema & & & & \\
Fragebogen zur Textverständlichkeit & - & $\mathrm{x}$ & - & - \\
\hline
\end{tabular}

\section{Testinstrumente}

Zur Erfassung des Lernerfolgs wurde ein selbstkonstruierter Fachwissenstest, der in einer Vorstudie auf Validität und Reliabilität getestet wurde (vgl. Autor 2017), eingesetzt. Der Test enthielt 23 Items im Multiple-Choice-Single-Select-Format. Das situationale Interesse wurde mithilfe von Subskalen aus dem Fragebogen nach Fechner (2009) direkt nach dem Lernen erhoben und bestand in dieser Studie aus Items, von denen sich die eine Hälfte auf das Interesse am Text und die andere Hälfte auf das Interesse am Thema bezogen. Die Items waren jeweils analog formuliert, nur das Wort „Text“ wurde durch das Wort „Thema“ ersetzt. Da die Textstruktur einen Einfluss auf das situationale Interesse nehmen kann (vgl. Schiefele 1996), wurden auch Items von Schraw (1997) zur Einschätzung der Textstruktur eingesetzt. Die Lernzeit kann eine mögliche Folge des situationalen Interesses sein (vgl. Schiefele und Krapp 1996) und wurde aus der Differenz der Anfangs- und der Endzeit beim Lernen mit der jeweiligen Textart erhoben. Die Schülerinnen und Schüler notierten auf Anweisung des Testleiters die Anfangszeit und selbstständig die Zeit nach dem Ende des Lernens mit dem jeweiligen Text. Anschließend wurde direkt das situationale Interesse erhoben. Danach konnten die Schülerinnen und Schüler eigenständig im Testheft weiterblättern und den Fachwissenstest bearbeiten. $\mathrm{Da}$ es bei den Lernmaterialien allgemein wichtig ist, die kognitive Beanspruchung nach Sweller et al. (2011) möglichst gering zu halten, wurde zusätzlich der Cognitive Load (Kalyuga et al. 1999; Paas 1992) begleitend nach dem Lesen der jeweiligen Textart miterhoben. Dazu wurde jeweils ein Rating-Item wie z.B. „Beim Lesen dieses Textes war meine geistige Anstrengung insgesamt ... " mit einer 7-stufigen Likert-Skala von „sehr gering “ (1) bis „sehr hoch“" (7) zum intrinsic, extrinsic und germane load implementiert. Für die Erhebung der Kontrollvariablen wurden ein Kognitiver-Fähigkeiten-Test (Heller und Perleth 2000), der FAM zur Erfassung der aktuellen Motivation in Lern- und Leistungssituationen (Rheinberg et al. 2001), der LGVT zur
Erfassung der Lesegeschwindigkeit und des Leseverständnisses (Schneider et al. 2007) sowie ein Fragebogen zur Lesemotivation (Schaffner 2009) verwendet. Letztere kann sich beispielsweise positiv auf das Textverstehen und auf die aktuelle Motivation auswirken. Aus zeitökonomischen Gründen wurden nur zwei Skalen, die objektbezogene und erlebnisbezogene Motivation, verwendet (vgl. Tab. 2).

\section{Stichprobenbeschreibung}

An der Studie nahmen insgesamt 677 Schülerinnen und Schüler aus 14 Klassen von sieben Gymnasien der 8. Jahrgangsstufe und elf Klassen der 8. und 9. Jahrgangsstufen von sechs Realschulen teil. Die teilnehmenden Schulen lagen sowohl in Ballungsgebieten als auch in Kleinstädten in Nordrhein-Westfalen. Die Studie wurde im regulären Chemieunterricht durchgeführt, sodass die Schülerinnen und Schüler noch kein Vorwissen zu den Alkalimetallen hatten. Da sich die Studie über drei Termine erstreckte, wurden Schülerinnen und Schüler, die mindestens einmal fehlten, aus der Analyse ausgeschlossen, sodass ein vollständiger Datensatz von insgesamt $N=510$ Schülerinnen und Schülern (weiblicher Anteil von 51,2\% und $M_{\text {Alter }}=13,6$, $S D=1,1)$ vorliegt. Da von manchen Schülerinnen und Schülern bei einigen Testinstrumenten keine Angaben gemacht wurden, variieren die Freiheitsgrade bei einigen Analysen leicht. Folgende Zuteilung zu den Gruppen kam durch die Randomisierung zustande (vgl. Tab. 3):

Deskriptiv zeigen sich leichte Unterschiede hinsichtlich des Geschlechts, der Chi-Quadrat-Test nach Pearson fiel aber nicht signifikant aus, sodass eine gleiche Ver-

Tab. 3 Überblick über die Zuteilung der Schülerinnen und Schüler auf Interventionsgruppen (Reschke 2017)

\begin{tabular}{llll}
\hline Interventionsgruppe & Geschlecht & & \\
\cline { 2 - 4 } & Weiblich & Männlich & Gesamt \\
\hline Geschichte (G) & 134 & 119 & 253 \\
Sachtext (S) & 127 & 130 & 257 \\
Gesamt & 261 & 249 & 510 \\
\hline
\end{tabular}


teilung angenommen werden kann. Zunächst wurden die Kontrollvariablen auf Mittelwertunterschiede zwischen den Gruppen anhand von $t$-Tests für unabhängige Stichproben überprüft. Die Ergebnisse dieser Tests zeigen, dass sich die Gruppen hinsichtlich des Fachinteresses $(\mathrm{S}>\mathrm{G}$ : $t(501,791)=2,136, p=0,033, d=0,189)$ und der erlebnisbezogenen Lesemotivation $(\mathrm{S}>\mathrm{G}: t(506)=2,357, p=0,019$, $d=0,209)$ unterscheiden. Somit werden diese beiden Variablen für weitere Berechnungen als Kovariate einbezogen. Die beiden Geschlechter unterscheiden sich - unabhängig von den Untersuchungsgruppen - beim Fachinteresse $\left(\sigma^{7}>\right.$ o : $\left.t(508)=5,132, p<0,001, d=0,455\right)$, bei der erlebnisbezogenen Lesemotivation $\left(\varphi>\sigma^{\prime}: t(504,372)=8,362\right.$, $p<0,001, d=0,742)$, bei den kognitiven Fähigkeiten ( $\left(+>\sigma^{7}\right.$ : $t(507)=2,228, p=0,026, d=0,197)$, beim Leseverständnis ( $\left.>>o^{\top}: t(501)=2,311, p=0,021, d=0,207\right)$, bei der objektbezogenen Lesemotivation $\left(q>\sigma^{\prime}: t(507)=2,115, p=0,035\right.$, $d=0,187$ ) und der Deutschnote ( $>\sigma^{\top}: t(507)=3,116$, $p=0,002, d=0,269)$. Für den Geschlechtervergleich werden diese Variablen hinsichtlich der Varianzanalysen als Kovariate einbezogen.

\section{Methodische Darlegung}

Neben $t$-Tests und Varianzanalysen wurden zudem einfache Regressionsmodelle sowie Mediatoranalysen per multipler Regression gerechnet. Durch einfache Regressionen kann geklärt werden, wie eine unabhängige Variable auf die abhängige Variable wirkt (direkter Effekt). Direkte Effekte sind Einflussbeziehungen, die nicht durch eine andere Variable beeinflusst werden (Urban und Mayerl 2011). In der Abb. 2 sind drei solcher Effekte allgemein anhand von $b_{z x}$ (Einfluss von $X$ auf $Z$ ), $b_{y x}$ (Einfluss von $X$ auf $Y$ ) und $b_{y z}$ (Einfluss von $\mathrm{Z}$ auf $\mathrm{Y}$ ) dargestellt.

Der direkte Effekt $b_{y x}$ kann durch $Z$ beeinflusst werden, sodass ein indirekter Effekt entsteht. Der indirekte Effekt lässt sich aus der Multiplikation der beiden direkten Effekte errechnen:

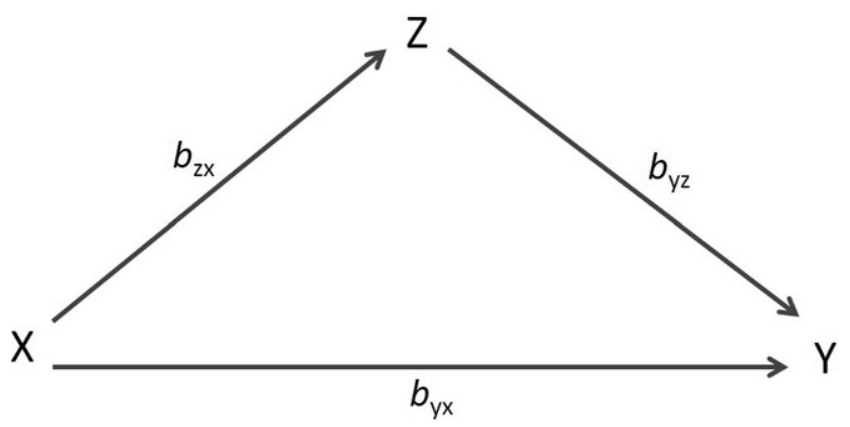

Abb. 2 Allgemeines Mediator-Modell mit direkten Effekten (partieller Mediationseffekt), dargestellt nach Urban und Mayerl (2011), nach Reschke (2017) bearbeitet

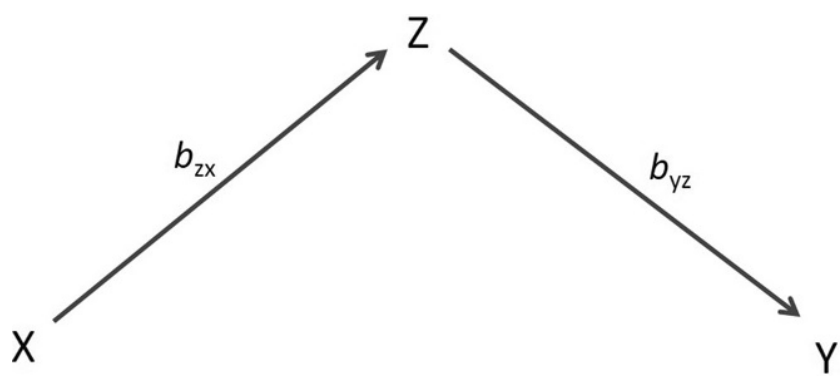

Abb. 3 Vollständiger Mediationseffekt, dargestellt nach Urban und Mayerl (2011), nach Reschke (2017) bearbeitet

\section{GI. 1: Berechnung des indirekten Effektes (nach Urban und Mayerl 2011)}

$b_{z x^{*} y z}=b_{z x} * b_{y z}$

Bei einem indirekten Effekt unterscheidet man zwischen einem partiellen und einem vollständigen Mediatoreffekt. Ein partieller Effekt liegt dann vor, wenn ein Effekt von X auf $\mathrm{Y}$ über $\mathrm{Z}$ mediiert wird und zugleich ein direkter Effekt von $X$ auf $Y$ vorliegt (siehe Abb. 2). Ein vollständiger Mediatoreffekt liegt hingegen dann vor, wenn ein Effekt von $\mathrm{X}$ auf $\mathrm{Y}$ komplett durch $\mathrm{Z}$ vermittelt wird und dadurch der direkte Effekt von X auf Y verschwindet (vgl. Abb. 3). Über einen Sobel-Test können Mediatoreffekte berechnet werden. Wirken bei einem Mediationsmodell zwei unabhängige Variablen auf die abhängige, so liegt eine doppelte Mediation vor. Die Mediationen können hierbei serien- oder parallelgeschaltet auf die abhängige Variable wirken (Baltes-Götz 2015). Solche doppelten Mediationen sind multiple Regressionen und wurden mit dem Programm „Process Version 2016“ (Hayes 2013) für SPSS berechnet. Die Passung des mediierten Modells wird über Schätzwerte - sogenannte Fitindizes - angegeben, die zusätzlich mit AMOS 23 (IBM) berechnet werden können. Hierbei werden bestimmte Werte berücksichtigt (vgl. Tab. 4). Wenn die Fitindizes gute Werte aufweisen, dann kann das Modell angenommen werden. Allerdings betont Byrne (2001) in diesem Zusammenhang, dass die Fitindizes keine absolute Auskunft über die Plausibilität eines Modells geben. Wichtiger ist es, dass das Modell anhand von theoretischen, statistischen und praktischen Überlegungen gerechtfertigt ist (Byrne 2001).

\section{Ergebnisse}

\section{Fachwissen}

Für den aus 19 Items bestehenden Fachwissenstests zeigt die Reliabilitätsanalyse zum Prä-Messzeitpunkt einen Cronbachs- $\alpha$-Wert von $0,29(M=0,38, S D=0,47)$, zum PostMesszeitpunkt einen Wert von 0,82 $(M=0,70, S D=0,44)$ 
Tab. 4 Theoretische Fitindizes zur Schätzung von Regressionsmodellen (Reschke 2017)

\begin{tabular}{llll}
\hline Benennung & Erläuterung & Toleranzbereich & Quelle \\
\hline CMIN/DF & $\begin{array}{l}\text { Minimum der Diskrepanzfunktion } \\
\text { geteilt durch Freiheitsgrade }\end{array}$ & $2 \leq$ CMIN/DF $\leq 5$ & Byrne (2001); Kline (2005) \\
CFI & Comparative Fit Index & CFI $>0,95$ & Hair et al. (1998) \\
TLI & Tucker-Lewis Index & Nahe 1 & Hair et al. (1998) \\
RMSEA & Root Mean Square Error of Approxi- & RMSEA $\leq 0,05$ (gut) & Browne und Cudeck (1993) \\
& mation & $0,05<$ RMSEA $\leq 0,08$ (akzeptabel) & \\
PCLOSE & $p$-Wert für RMSEA & RMSEA $>0,08$ (inakzeptabel) & Browne und Cudeck (1993) \\
\hline
\end{tabular}

Tab. 5 Mittelwerte zu Interventionsgruppen im Fachwissenstest bei allen Messzeitpunkten (Reschke 2017)

\begin{tabular}{|c|c|c|c|c|c|c|c|c|c|}
\hline \multirow[t]{2}{*}{ Gruppe } & \multicolumn{3}{|c|}{ Prä-Test } & \multicolumn{3}{|c|}{ Post-Test } & \multicolumn{3}{|c|}{ Follow-up-Test } \\
\hline & $\bar{n}$ & $M(S D)$ & $95 \% \mathrm{CI}$ & $n$ & $M(S D)$ & $95 \% \mathrm{CI}$ & $\bar{n}$ & $M(S D)$ & $95 \% \mathrm{CI}$ \\
\hline$\overline{\mathrm{G}}$ & 253 & $7,11(2,45)$ & {$[6,81,7,42]$} & 253 & $13,98(4,14)$ & {$[13,46,14,49]$} & 253 & $11,65(4,03)$ & {$[11,15,12,15]$} \\
\hline$S$ & 257 & $7,45(2,39)$ & {$[7,15,7,74]$} & 257 & $12,71(4,05)$ & {$[12,21,13,21]$} & 257 & $10,91(3,87)$ & {$[10,44,11,39]$} \\
\hline
\end{tabular}

$n$ Teilstichprobe, $M$ Mittelwerte, $S D$ Standardabweichung, $C I$ Konfidenzintervall

und zum Follow-up-Messzeitpunkt einen Wert von 0,76 $(M=0,59, S D=0,47)$. Für die letzten Messzeitpunkte liegen gute bis sehr gute Werte vor, nur für den Prä-Messzeitpunkt ist der Wert nicht zufriedenstellend, dies ist aber dem geschuldet, dass die Schülerinnen und Schüler zu diesem Zeitpunkt mangels Vorwissen geraten haben. In der Tab. 5 sind die erreichten Mittelwerte beider Gruppen zu allen Messzeitpunkten dargestellt:

Deskriptiv fällt auf, dass die Schülerinnen und Schüler in beiden Gruppen durch die Intervention ihre Punktzahl im Fachwissenstest ungefähr verdoppeln und die Punktzahl zum Follow-up-Messzeitpunkt leicht abnimmt. Zudem unterscheiden sich die beiden Gruppen zum Prä-Messzeitpunkt kaum, während sich deskriptive Unterschiede bei den letzten beiden Messzeitpunkten erkennen lassen. Dort erreicht die Geschichtsgruppe deskriptiv höhere Mittelwerte als die Sachtextgruppe. Ergebnisse der ANCOVA mit Messwiederholung und dem Fachinteresse sowie der erlebnisbezogenen Lesemotivation als Kovariaten zeigen, dass sich die Gruppen hinsichtlich ihrer Mittelwerte unterscheiden (Haupteffekt der Gruppe: $F(1,506)=9,329, p=0,003$, $\left.\eta_{p}{ }^{2}=0,018\right)$. Zudem liegt ein Haupteffekt des Messzeitpunktes vor $\left(F(1,917,966,099)=6,198, p=0,002, \eta_{p}{ }^{2}=0,012\right.$, Greenhouse-Geisser korrigert mit $\varepsilon=0,958)$. Innerhalb der Gruppen unterscheiden sich die erreichten Mittelwerte zu den drei Messzeitpunkten (Interaktionseffekt von Gruppe * Messzeitpunkt: $F(1,916,965,808)=14,487, p<0,001$, $\eta_{p}{ }^{2}=0,028$, Greenhouse-Geiser korrigiert: $\varepsilon=0,958$ ). Vergleicht man die Mittelwerte beider Gruppen zu jedem Messzeitpunkt einzeln, so zeigen die ANCOVAs, dass sich die Mittelwerte der Gruppen zum Post- und Followup-Messzeitpunkt signifikant unterscheiden (post: $F(1$, 506) $=17,828, p=0,001, \eta_{p}{ }^{2}=0,034$ und follow up: $F(1$,
506) $\left.=8,214, p=0,004, \eta_{p}{ }^{2}=0,016\right)$, jedoch nicht zum PräMesszeitpunkt $(F(1,506)=1,533, p=0,216)$ (vgl. Abb. 4).

Rechnet man das Geschlecht zusätzlich als Zwischenfaktor und die Kovariaten bei den Geschlechterunterschieden (siehe Stichprobenbeschreibung) mit ein, so zeigt das Ergebnis der ANCOVA, dass der Gruppeneffekt bestehen bleibt (Haupteffekt der Gruppe: $F(1,508)=13,453$, $\left.p<0,001, \eta_{p}{ }^{2}=0,027\right)$. Schaut man sich den durchschnittlichen Summenscore zum Post-Messzeitpunkt getrennt nach den Geschlechtern an, so ist der Effekt zwischen beiden Gruppen bei den Mädchen größer $(F(1,258)=14,692$, $\left.p<0,001, \eta_{p}{ }^{2}=0,054\right)$ als bei den Jungen $(F(1,246)=4,017$, $\left.p=0,046, \eta_{p}{ }^{2}=0,016\right)$. Bei den Mädchen liegt ein mittlerer Effekt und bei den Jungen ein kleiner Effekt vor. Betrachtet man den Lernzuwachs, also die Differenz der erreichten Mittelwerte zum Post- und Prä-Messzeitpunkt, erreicht die Geschichtsgruppe $(M=6,86, S D=4,37,95 \%$ CI $[6,32$, $7,40])$ deskriptiv ca. 1,5 Punkte mehr als die Sachtextgruppe $(M=5,26, S D=3,87,95 \%$ CI $[4,78,5,74])$. Das Ergebnis der ANCOVA zeigt zudem, dass dieser Mittel-

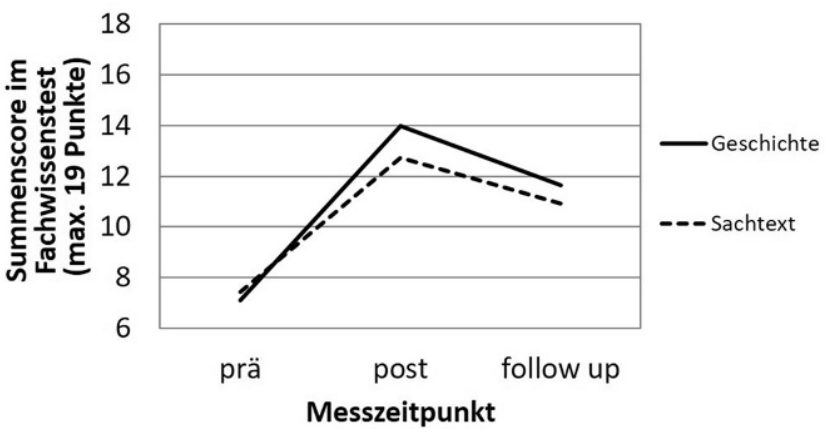

Abb. 4 Verläufe der Mittelwerte beider Interventionsgruppen zu allen Messezeitpunkten im Fachwissenstest nach Reschke (2017) bearbeitet 
Abb. 5 Vergleich der Gruppen beim Lernzuwachs hinsichtlich des Geschlechts nach Reschke (2017)

Abb. 6 Gruppen-/Geschlechtervergleich beim textbezogenen situationalen Interesse nach Reschke (2017)
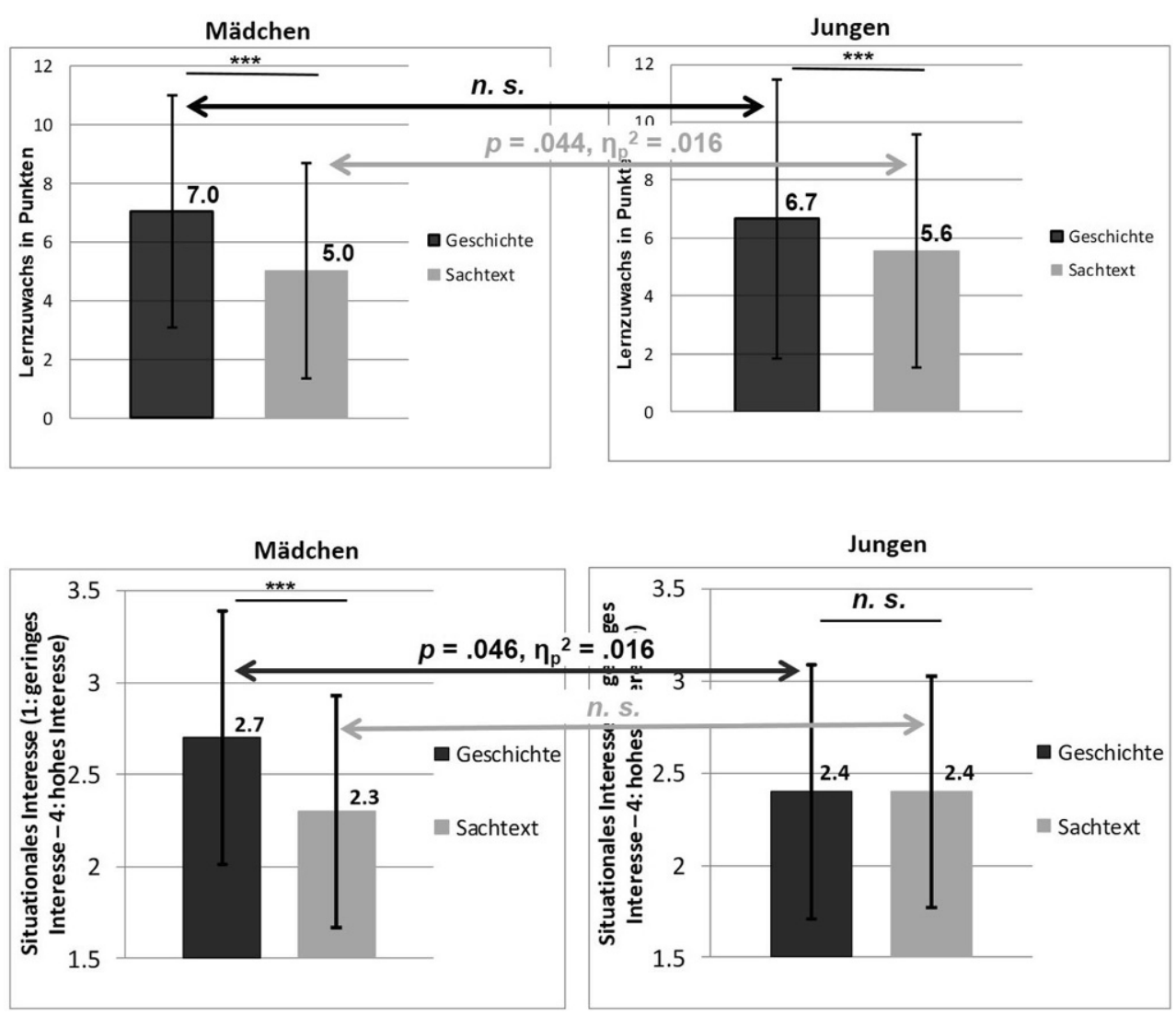

wertunterschied signifikant ist und einem mittleren Effekt entspricht $\left(F(1,506)=23,856, p<0,001, \eta_{p}{ }^{2}=0,045\right)$. Betrachtet man auch hier die Geschlechter getrennt, so zeigt sich, dass der Gruppenunterschied im Lernzuwachs bei den Mädchen deutlich größer ist als bei den Jungen und mit einem größeren Effekt einhergeht ( $($ : $F(1,258)=22,425$, $p<0,001, \eta_{p}{ }^{2}=0,081$ und $\sigma^{x}: F(1,246)=5,514, p=0,020$, $\left.\eta_{p}{ }^{2}=0,022\right)$. In der Abb. 5 ist dies veranschaulicht.

Darüber hinaus lässt sich an der Abbildung erkennen, dass sich die Geschlechter innerhalb der Sachtext-Gruppe signifikant unterscheiden. Jungen erreichen mit dem Sachtext im Vergleich zu den Mädchen einen signifikant höheren Lernzuwachs mit einer kleinen Effektstärke (ANCOVA: $\left.F(1,249)=4,853, p=0,044, \eta_{p}{ }^{2}=0,016\right)$. Innerhalb der Geschichts-Gruppe unterscheiden sich die Geschlechter hinsichtlich des Lernzuwachses jedoch nicht signifikant.

\section{Situationales Interesse}

Eine Hauptkomponentennalyse mit Varimax-Rotation zeigt, dass sich die Items mit Ausnahme eines Items den zwei Skalen ,textbezogen“ und ,thematisch“ eindeutig zuordnen lassen. Die Reliabilitätsanalysen für beide Skalen zeigt einen Cronbachs- $\alpha$-Wert von 0,91 $(M=2,41, S D=0,90)$. Die

Mittelwerte beider Skalen zeigen, dass die Schülerinnen und Schüler in beiden Gruppen deskriptiv ein mittleres thematisches und textbezogenes Interesse haben (vgl. Tab. 6).

Die Ergebnisse der ANCOVA mit der Gruppe als Zwischensubjektfaktor sowie dem Fachinteresse und der erlebnisbezogenen Lesemotivation als Kovariate zeigen, dass sich die Mittelwerte der Gruppen hinsichtlich des textbezogenen Interesses signifikant mit einem kleinen Effekt unterscheiden $\left(F(1,505)=10,856, p=0,001, \eta_{p}{ }^{2}=0,021\right)$. Betrachtet man die Geschlechter, sind die Mädchen stärker an der Geschichte interessiert als am Sachtext (vgl. Abb. 6). Der Unterschied geht mit einem mittleren Ef-
Tab. 6 Mittelwerte der Interventionsgruppen zu beiden Skalen des situationalen Interesses (Reschke 2017)

\begin{tabular}{|c|c|c|c|c|c|c|}
\hline \multicolumn{4}{|c|}{ Gruppen Textbezogenes Interesse } & \multicolumn{3}{|c|}{ Thematisches Interesse } \\
\hline & $\bar{n}$ & $M(S D)$ & $95 \% \mathrm{CI}$ & $n$ & $M(S D)$ & $95 \% \mathrm{CI}$ \\
\hline $\mathrm{G}$ & 251 & $2,55(0,77)$ & {$[2,46,2,65]$} & 251 & $2,42(0,71)$ & {$[2,33,2,50]$} \\
\hline$S$ & 257 & $2,41(0,88)$ & {$[2,33,2,49]$} & 257 & $2,36(0,69)$ & {$[2,28,2,45]$} \\
\hline
\end{tabular}

$n$ Teilstichprobe, $M$ Mittelwerte, $S D$ Standardabweichung, $C I$ Konfidenzintervall 
Tab. 7 Mittelwerte beider Geschlechter zur Lernzeit (Reschke 2017)

\begin{tabular}{|c|c|c|c|c|c|c|}
\hline \multirow[t]{2}{*}{ Geschlecht } & \multicolumn{3}{|c|}{ Geschichte } & \multicolumn{3}{|c|}{ Sachtext } \\
\hline & $\bar{n}$ & $M(S D)$ & $95 \% \mathrm{CI}$ & $n$ & $M(S D)$ & $95 \% \mathrm{CI}$ \\
\hline Mädchen & 131 & $13,47(3,46)$ & {$[12,87,14,06]$} & 125 & $7,17(3,64)$ & {$[6,52,7,81]$} \\
\hline Jungen & 116 & $12,43(3,38)$ & {$[11,81,13,05]$} & 124 & $6,10(2,97)$ & {$[5,57,6,62]$} \\
\hline
\end{tabular}

$n$ Teilstichprobe, $M$ Mittelwerte, $S D$ Standardabweichung, $C I$ Konfidenzintervall fekt einher $\left(F(1,258)=16,821, p<0,001, \eta_{p}{ }^{2}=0,062\right)$. Bei den Jungen gibt es keine Unterschiede zwischen dem Interesse an der Geschichte und am Sachtext. Schaut man sich das textbezogene situationale Interesse innerhalb der Geschichts-Gruppe an (vgl. schwarzer Pfeil in Abb. 6), so zeigt sich bei den Mädchen ein signifikant höheres textbezogenes situationales Interesse als bei den Jungen $(t(227,014)=2,381, p=0,018, d=0,312)$. Auch unter Einbeziehung der Kovariaten bleibt der Effekt vorhanden $\left(F(1,245)=4,009, p=0,046, \eta_{p}{ }^{2}=0,016\right)$. Innerhalb der Sachtext-Gruppe zeigen sich bezüglichdes textbezogenen situationalen Interesses keine Geschlechterunterschiede (vgl. grauer Pfeil in Abb. 6).

Hinsichtlich des thematischen situationalen Interesses zeigt das Ergebnis der ANCOVA, dass sich die Mittelwerte der beiden Gruppen signifikant unterscheiden $(F(1$, 504) $\left.=4,050, p=0,045, \eta_{p}{ }^{2}=0,008\right)$. Dieser Effekt ist allerdings sehr klein und kommt nur durch die Mädchen zustande. Die Mädchen, die mit der Geschichte lernen, zeigen ein höheres thematisches situationales Interesse als die Mädchen, die mit dem Sachtext lernen $(F(1,258)=4,470$, $\left.p=0,035, \eta_{p}{ }^{2}=0,017\right)$. Die Effektstärke ist allerdings gering. Bei den Jungen gibt es zwischen den Gruppen keinen Unterschied hinsichtlich des thematischen situationalen Interesses. Vergleicht man die Geschlechter innerhalb der Textsorten-Gruppen, so zeigen sich keine Geschlechterunterschiede beim thematischen Interesse. Das thematische situationale Interesse korreliert insgesamt sehr stark mit dem textbezogenen situationalen Interesse $(r=0,751$, $p<0,001)$.

\section{Lernzeit}

Insgesamt haben 14 Schülerinnen und Schüler keine Angaben bei der Anfangszeit und/oder der Endzeit gemacht, sodass diese für die Analyse herausgenommen wurden. Die Schülerinnen und Schüler konnten selbst entscheiden, wann ihr Lernprozess beendet war. Diejenigen, die mit der Geschichte lernen, lernen fast doppelt so lange wie jene mit dem Sachtext (G: $M=12,98, S D=3,46,95 \%$ CI $[12,55$; $13,41]$ und $S: M=6,63, S D=3,36,95 \%$ CI $[6,21 ; 7,05])$. Das Ergebnis der ANCOVA zeigt, dass diese Unterschiede hoch signifikant sind und mit einem großen Effekt einhergehen $\left(F(1,493)=420,311, p<0,001, \eta_{p}{ }^{2}=0,462\right)$. Hinsichtlich des Geschlechts zeigt sich in beiden Gruppen, dass die Mädchen im Durchschnitt eine Minute länger mit dem jeweiligen Text lernen als die Jungen (vgl. Tab. 7).

Die Ergebnisse der ANCOVA zeigen, dass sowohl die Mädchen als auch die Jungen mit der Geschichte länger lernen. Dies geht hoch signifikant mit einem starken Effekt einher (Mädchen: $F(1,253)=197,936, p<0,001$, $\eta_{p}{ }^{2}=0,441$; Jungen: $F(1,238)=227,598, p<0,001, \eta_{p}{ }^{2}=$ 0,491). Ergebnisse der ANCOVA für den Geschlechtervergleich innerhalb einer Gruppe zeigen, dass sich die Mittelwerte der Geschlechter in beiden Gruppen hoch signifikant mit einem kleinen bis mittleren Effekt unterscheiden $\left(\mathrm{G}: F(1,247)=12,222, p=0,001, \eta_{p}{ }^{2}=0,050\right.$ und S: $\left.F(1,247)=5,580, p=0,019, \eta_{p}{ }^{2}=0,023\right)$. In der Abb. 7 sind die Lernzeiten getrennt nach den Geschlechtern und Gruppen dargestellt.
Abb. 7 Gruppen/-Geschlechtervergleich bei der tatsächlichen Lernzeit (Reschke 2017)

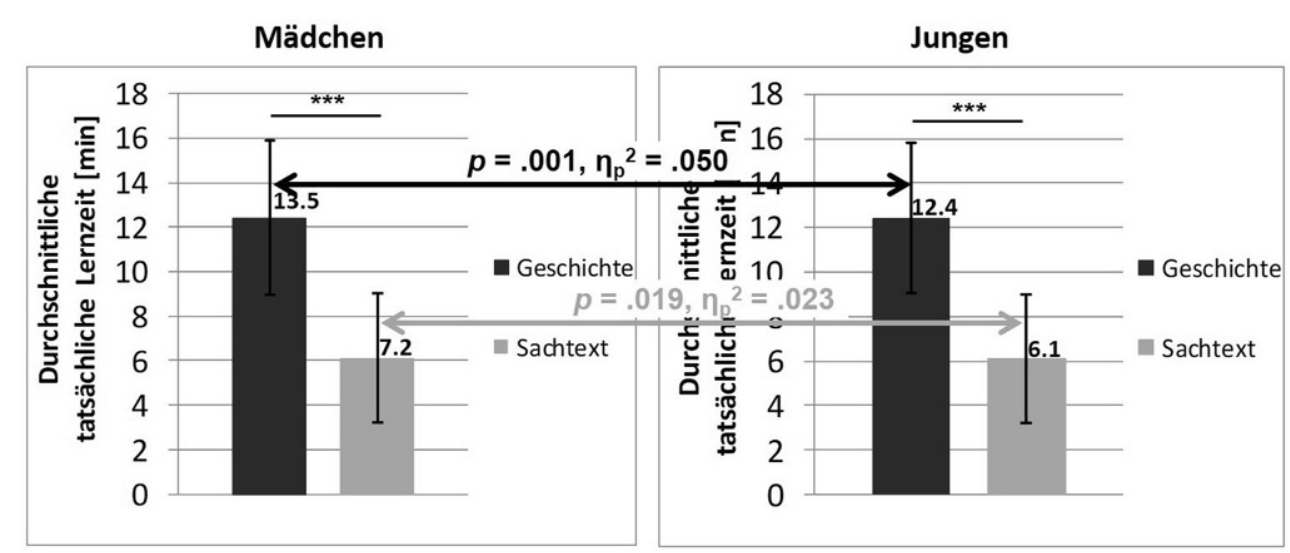




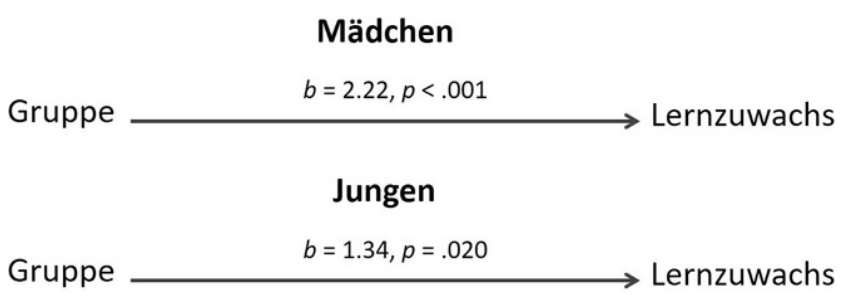

Abb. 8 Regressionsmodell mit der Wirkung der Gruppe als unabhängige Variable auf den Lernzuwachs als abhängige Variable für beide Geschlechter; unstandardisierte Koeffizienten (Reschke 2017)

\section{Zusammenhang zwischen dem situationalen Interesse, der Lernzeit und dem Lernerfolg}

Das Ergebnis einer einfachen Regression mit unstandardisierten Koeffizienten $(b)$ und unter Einbeziehung der Kovariaten - Fachinteresse und erlebnisbezogene Lesemotivation - zeigt den Einfluss der Gruppe auf den Lernzuwachs getrennt nach den Geschlechtern in der Abb. 8. Die Gruppenvariable wurde hierbei mit 0 für die Sachtext-Gruppe und mit 1 für die Geschichts-Gruppe codiert. An den Werten ist zu erkennen, dass der Einfluss der Gruppe auf den Lernzuwachs bei den Mädchen höher ist als bei den Jungen.

Schaut man sich die Korrelationen an, so korreliert der Lernzuwachs mit dem textbezogenen situationalen Interesse $(r=0,350, p \leq 0,01)$ und mit der Lernzeit $(r=0,254$, $p \leq 0,01)$ sowie die Lernzeit mit dem textbezogenen situationalen Interesse $(r=0,195, p \leq 0,01)$. Da sich die Untersuchungsgruppen hinsichtlich des textbezogenen Interesses, der Lernzeit und des Lernzuwachses unterscheiden, wurden Mediationsmodelle gerechnet. Hierbei wurden die Analysen getrennt nach Geschlechtern vorgenommen, da Geschlechtereffekte auftreten. Zunächst wurde der Einfluss der Gruppe auf den Lernzuwachs über das textbezogene situationale Interesse überprüft. Das Ergebnis der Analyse macht deutlich, dass bei den Mädchen ein hoch signifikanter Einfluss der Gruppe auf das textbezogene situationale Interesse vorhanden ist (Abb. 9). Da der Regressionskoeffizient von $b=2,22$ auf $b=1,72$ sinkt und dennoch signifikant vorhanden bleibt, wird der direkte Einfluss der

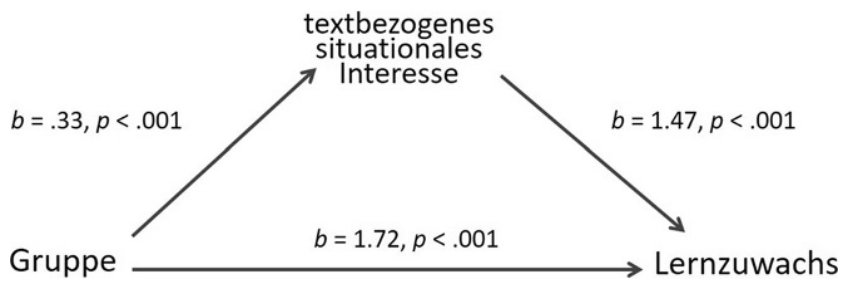

Abb. 9 Mediationsmodell für unstandardisierte Koeffizienten der partiellen Mediation des textbezogenen situationalen Interesses als Mediator über die Gruppe auf den Lernzuwachs unter Kontrolle der Kovariaten Fachinteresse und erlebnisbezogene Lesemotivation; mit einem BCa bootstrapped Konfidenzintervall (CI), basiert auf 1000 Samples, Modell 4; Mädchen (Reschke 2017)

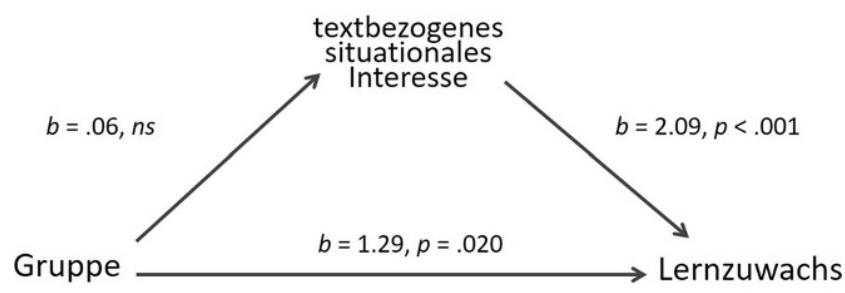

Abb. 10 Nicht vorhandene Mediation für unstandardisierte Koeffizienten des textbezogenen situationalen Interesses als Mediator über die Gruppe auf den Lernzuwachs unter Kontrolle der Kovariaten Fachinteresse und erlebnisbezogene Lesemotivation; mit einem BCa bootstrapped Konfidenzintervall (CI), basiert auf 1000 Samples, Modell 4; Jungen (Reschke 2017)

Gruppe auf den Lernzuwachs partiell über das textbezogene situationale Interesse mediiert. Daher liegt eine partielle Mediation durch das textbezogene situationale Interesse bei den Mädchen vor.

Bei den Jungen zeigt sich hingegen kein signifikanter Einfluss der Gruppe auf das textbezogene situationale Interesse (vgl. Abb. 10). Dadurch nimmt der Regressionskoeffizient nicht ab, sodass der direkte Einfluss der Gruppe auf den Lernzuwachs nicht über das textbezogene situationale Interesse mediiert wird (vgl. Abb. 10).

Schaut man sich den Einfluss der Gruppe auf den Lernzuwachs über die Lernzeit an, so wird deutlich, dass das Mediationsmodell für beide Geschlechter mit gleich starken Effekten gültig ist. Daher werden die Werte für die Gesamtstichprobe dargestellt (vgl. Abb. 11).

Bei diesem Mediationsmodell liegt ein hoch signifikanter Einfluss der Gruppe auf die Lernzeit vor. Der direkte Einfluss der Gruppe auf den Lernzuwachs verschwindet durch die Lernzeit vollständig, da der Wert für den Regressionskoeffizienten $b=0,37$ des direkten Einflusses nicht mehr signifikant vorhanden ist. Dadurch liegt eine vollständige Mediation vor (Abb. 11).

Da bei den Mädchen die Gruppe sowohl über das textbezogene situationale Interesse als auch über die Lernzeit auf den Lernzuwachs wirkt, kann es sein, dass die beiden Mediationen nicht einzeln, sondern zusammen auf

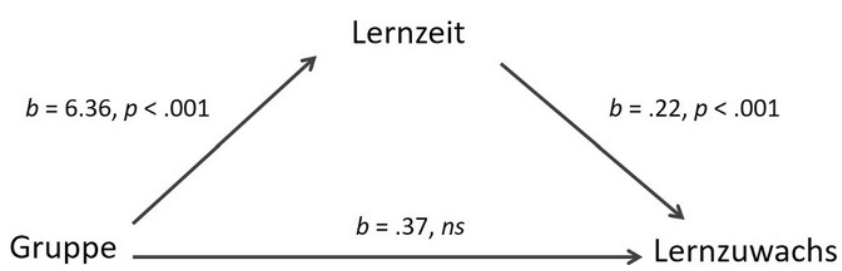

Abb. 11 Mediationsmodell für unstandardisierte Koeffizienten der vollständigen Mediation der Lernzeit als Mediator über die Gruppe auf den Lernzuwachs unter Kontrolle der Kovariaten Fachinteresse und erlebnisbezogene Lesemotivation; mit einem BCa bootstrapped Konfidenzintervall (CI), basiert auf 1000 Samples, Modell 4 (Reschke 2017) 


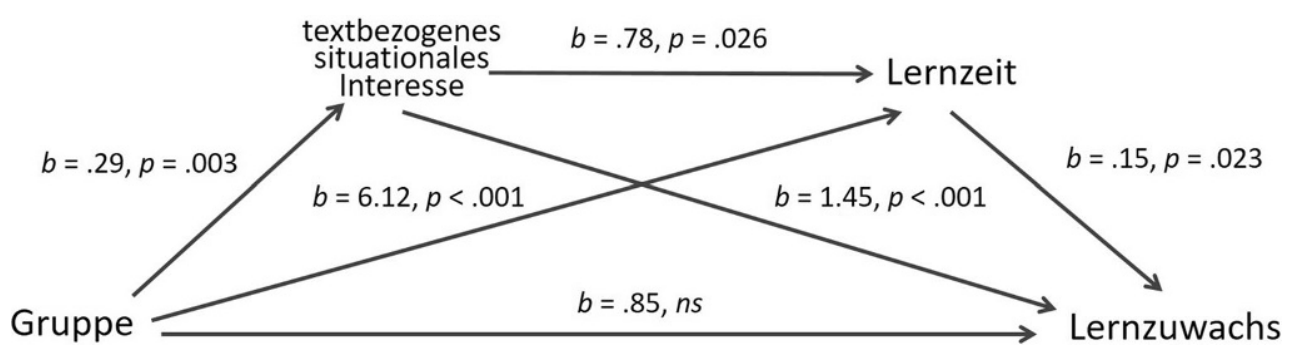

Abb. 12 Multiples Regressionsmodell für unstandardisierte Koeffizienten von Gruppe als Prädiktor für den Lernzuwachs, mediiert über das textbezogene situationale Interesse und die Lernzeit als in seriengeschaltete Mediatoren unter Kontrolle der Kovariaten Fachinteresse und erlebnisbezogene Lesemotivation; mit einem BCa bootstrapped Konfidenzintervall (CI), basiert auf 10.000 Samples, Modell 6; Mädchen (Reschke 2017)

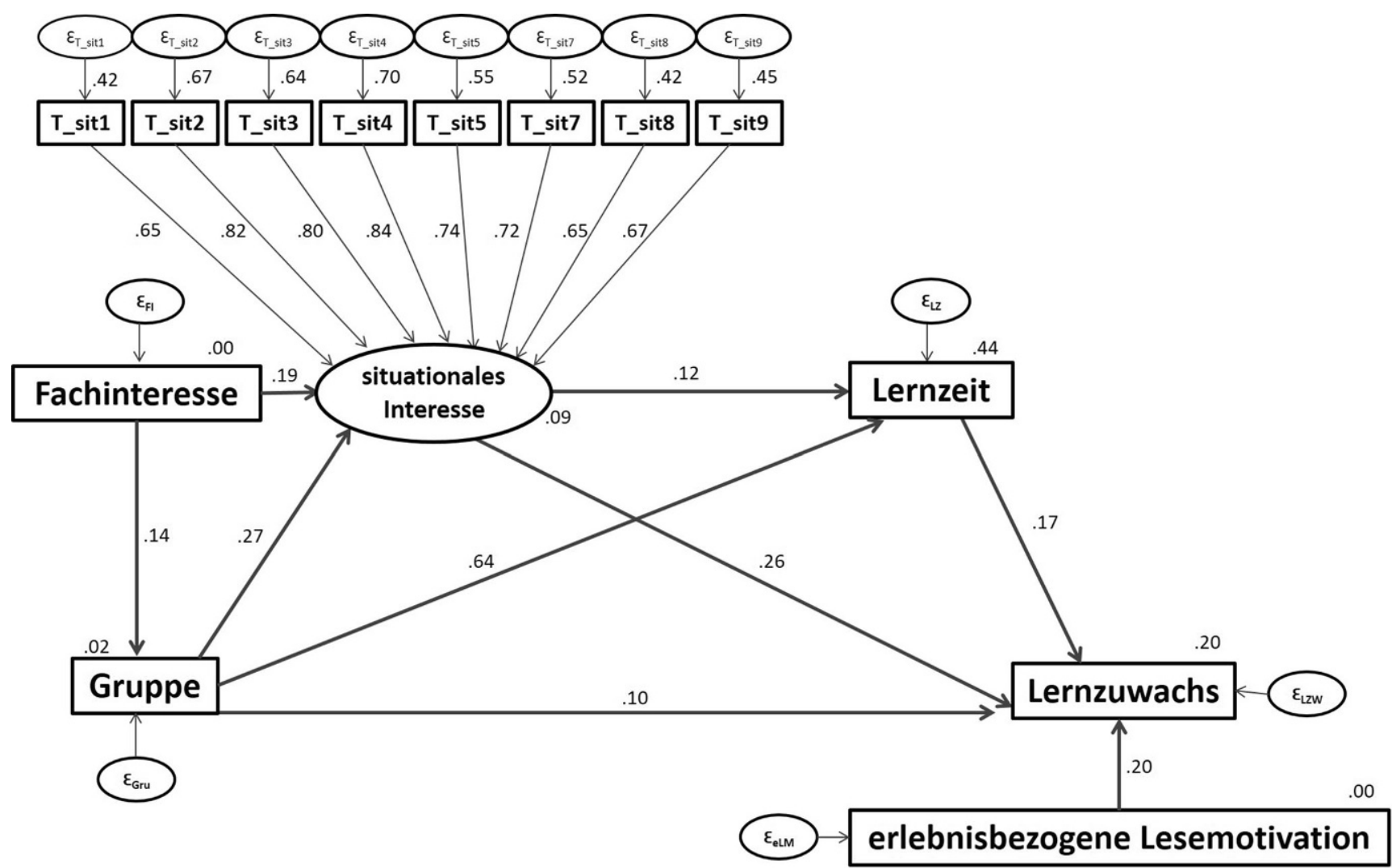

Abb. 13 Gesamtmodell für die Mädchen zur Aufklärung des Lernzuwachses; mit SPSS Amos geschätzt (Reschke 2017)

den Lernzuwachs wirken. Die partielle Korrelation zwischen dem textbezogenen situationalen Interesse und der Lernzeit in Abhängigkeit von der Gruppe gibt einen ersten Hinweis auf das Zusammenwirken der beiden Mediationen auf den Lernzuwachs, da diese hoch signifikant vorhanden ist $\left(\mathrm{r}_{\text {(situationales Interesse, Lernzeit),Gruppe }}=0,185, p<0,001\right)$. Aus diesem Grund werden beide Meditationen in einem multiplen Regressionsmodell auf deren Wechselwirken überprüft. Das Modell ist in Abb. 12 dargestellt. An dem Modell wird deutlich, dass sowohl das textbezogene situationale Interesse als auch die Lernzeit über die Gruppe einzeln auf den Lernzuwachs wirken, aber auch miteinander wechselwirken. Die Gruppe wirkt auch über das textbezogene situationale Inte- resse auf die Lernzeit. Der Einfluss der Gruppe wird daher insgesamt über das textbezogene Interesse sowie die Lernzeit mediiert, sodass der direkte Einfluss der Gruppe auf den Lernzuwachs verschwindet. Der Regressionskoeffizient $b$ des direkten Einflusses wird nicht mehr signifikant. Insgesamt werden für das multiple Regressionsmodell $20 \%$ an Varianz des Lernzuwaches aufgeklärt.

Das Strukturmodell wurde zudem einmal mit Amos 23 (Arbuckle 2015) gerechnet, um die Passung des multiplen Mediationsmodells schätzen zu lassen, da das Programm „Process“ eine solche Schätzung nicht liefert. Da es sich beim textbezogenen situationalen Interesse um eine latente Variable handelt und Amos 23 die Modellierung latenter 

werte für das Regressionsmodell (Reschke 2017)
Tab. 8 Überblick über die Fit-

\begin{tabular}{ll}
\hline Fitbezeichnung & Fitwerte \\
\hline CMIN & 258,806 \\
DF & 122 \\
CMIN/DF & 2,12 \\
Signifikanz & $<0,001$ \\
CFI & 0,952 \\
TLI & 0,928 \\
RMSEA & 0,047 \\
PCLOSE & 0,725 \\
\hline
\end{tabular}

Variablen zulässt, wurde dieses direkt über die einzelnen Items in das Modell einbezogen (Abb. 13). Ergebnisse dieser Analyse zeigen Fitwerte für dieses Modell in einem guten Bereich (vgl. Tab. 8), sodass eine gute Passung des Modells angenommen werden kann.

\section{Zusammenfassung und Diskussion der Ergebnisse}

Die Ergebnisse dieser Studie zeigen, dass die Schülerinnen und Schüler mit beiden Texten signifikante Lernzuwächse erreichen. Hinsichtlich der ersten Forschungsfrage lässt sich zeigen, dass die Schülerinnen und Schüler, die mit der Geschichte lernen, einen signifikant höheren Lernzuwachs erreichen als die Schülerinnen und Schüler, die mit dem Sachtext lernen. Das erlernte Fachwissen ist bei den Schülerinnen und Schülern nach vier bis sechs Wochen immer noch vorhanden. Diejenigen, die mit der Geschichte lernen, haben dann immer noch ein höheres Fachwissen als diejenigen, die mit dem Sachtext lernen. Insgesamt liegt ein mittlerer Haupteffekt für das textbezogene und das thematische situationale Interesse vor (Forschungsfrage 2). Das textbezogene situationale und das thematische situationale Interesse korrelieren insgesamt stark miteinander. Hinsichtlich der fünften Forschungsfrage kann man festhalten, dass beide Haupteffekte nur durch die Mädchen entstehen. Die Mädchen sind stärker an der Geschichte interessiert als an dem Sachtext. Außerdem sind die Mädchen im Vergleich zu den Jungen innerhalb der Geschichts-Gruppe auch stärker an der Geschichte interessiert. Ein ähnlicher Geschlechtereffekt konnte in der Studie von Martensen et al. (2007) nachgewiesen werden. Daraus kann man schlussfolgern, dass die Mädchen mit Lesegeschichten besonders gefördert werden. Bei den Jungen hingegen macht es keinen Unterschied, ob sie einen Sachtext oder eine Lesegeschichte bekommen. Vielleicht könnte die Sprachfähigkeit einen entscheidenden Einfluss darauf nehmen. Denn Mädchen haben allgemein eine höhere Sprachfähigkeit als die Jungen (vgl. Martiny und Fröhlich 2020), sodass sich das bei den Jungen negativ auf das Interesse an der Lesegeschichte auswirken könnte.
Hinsichtlich der Lernzeit (Forschungsfrage 3) zeigt sich, dass beide Geschlechter mit der Geschichte länger lernen. Es liegt zwar nahe, dass der Effekt durch die längere Textlänge der Geschichte zustande kommt, dennoch war es den Schülerinnen und Schüler (vgl. Abschn. Entwicklung der Lernmaterialien) zu jederzeit gestattet, das Lesen und damit den eigenen Lernprozess zu beenden oder den Text mehrfach zu lesen. Aus diesem Grund wurde das situationale Interesse miterhoben, um zu schauen, ob dieses einen Einfluss auf das Lernen und damit die Lernzeit nimmt. Hinsichtlich dieses Einflusses veranschaulicht das multiple Regressionsmodell, dass ein kausaler Zusammenhang zwischen dem Interesse an der Geschichte, der Lernzeit und dem Lernzuwachs unter Einbeziehung des Fachinteresses und der erlebnisbezogenen Lesemotivation als Kovariaten nur für die Mädchen vorhanden ist. Daraus lässt sich ableiten, dass die höhere Lernzeit nicht durch die Textlänge zustande kommt, sondern durch das höhere Interesse an der Lesegeschichte. Hinsichtlich der Forschungsfrage 5 lässt sich daher festhalten, dass bei den Mädchen die Textsorte zugunsten der neuen Geschichte einen Einfluss auf das situationale textbezogene Interesse, die Lernzeit und den Lernzuwachs nimmt. Je höher also das textbezogene situationale Interesse bei den Mädchen ist, desto höher ist ihre Lernzeit und desto höher wiederum der Lernzuwachs. Für dieses Modell liegen gute Passungswerte vor. Insgesamt werden $20 \%$ an Varianz für den Lernzuwachs bei den Mädchen aufgeklärt. Es kann daher festgehalten werden, dass die Interessantheit der Geschichte sich positiv auf das situationale Interesse der Mädchen auswirkt. Bei den Jungen hat nur die Lernzeit einen Einfluss auf den Lernzuwachs. Naheliegende Einflüsse wie beispielsweise das Fachinteresse oder die Chemienote können bei den Jungen hinsichtlich der Lernzeit keine Effekte aufklären. Aus diesem Grund bleibt für die Jungen weiterer Aufklärungsbedarf bezüglich möglicher Einflussfaktoren auf die Lernzeit (Forschungsfrage 3 und 5), denn auch diese hätten jederzeit das Lernen selbstständig beenden können. Die Kontrollvariablen ,aktuelle Motivation“ und „kognitive Fähigkeiten“ sowie die Prozessvariable „Cognitive Load" nehmen keinen Einfluss auf den Lernerfolg.

Die in dieser Arbeit entwickelte und untersuchte Lesegeschichte stellt somit eine geeignete Möglichkeit für einen Einsatz im Chemieunterricht dar. Dies gilt besonders zur Förderung des Lernerfolgs bei den Jungen und Mädchen sowie zur Förderung des situationalen Interesses der Mädchen. Aufbauend auf dieser Arbeit könnten daher weitere Lesegeschichten zu anderen chemischen Schulthemen entwickelt und eingesetzt werden, sodass diese Textart eine Alternative zu einem herkömmlichen Schulbuchtext sein kann.

Abschließend lässt sich festhalten, dass die entwickelte und untersuchte Lesegeschichte eine geeignete Möglichkeit für einen Einsatz im Chemieunterricht darstellt, weil sie den 
Lernprozess der Mädchen und Jungen positiv zu begünstigen scheint und bei den Mädchen sogar das situationale Interesse fördert.

\section{Downloads}

- Lesegeschichte: https://drive.google.com/file/d/1zWPSE hwKAfHfniEYhCibPPSWTsdQg4vM/view?usp=sharing

- Sachtext: https://drive.google.com/file/d/1N1lKwrXRH2 K6BUZ8PJZkJKs4yas_hioY/view?usp=sharing

- Fachwissenstest: https://drive.google.com/file/d/1UlNo VkQMcnuWEKPoE35lVZElX88MEZGb/view?usp=sha ring

Danksagung Wir bedanken uns herzlich bei den Schülerinnen und Schüler, den Lehrkräften und den Schulleitungen der beteiligten Schulen; ohne ihre Unterstützung wäre die Durchführung dieser Studie nicht möglich gewesen. Des Weiteren bedanken wir uns bei der Stiftung Mercator für die Finanzierung des Projektes Ganz In (08-341), in dessen Rahmen die Studie stattgefunden hat.

Funding Open Access funding enabled and organized by Projekt DEAL.

Open Access Dieser Artikel wird unter der Creative Commons Namensnennung 4.0 International Lizenz veröffentlicht, welche die Nutzung, Vervielfältigung, Bearbeitung, Verbreitung und Wiedergabe in jeglichem Medium und Format erlaubt, sofern Sie den/die ursprünglichen Autor(en) und die Quelle ordnungsgemäß nennen, einen Link zur Creative Commons Lizenz beifügen und angeben, ob Änderungen vorgenommen wurden.

Die in diesem Artikel enthaltenen Bilder und sonstiges Drittmaterial unterliegen ebenfalls der genannten Creative Commons Lizenz, sofern sich aus der Abbildungslegende nichts anderes ergibt. Sofern das betreffende Material nicht unter der genannten Creative Commons Lizenz steht und die betreffende Handlung nicht nach gesetzlichen Vorschriften erlaubt ist, ist für die oben aufgeführten Weiterverwendungen des Materials die Einwilligung des jeweiligen Rechteinhabers einzuholen.

Weitere Details zur Lizenz entnehmen Sie bitte der Lizenzinformation auf http://creativecommons.org/licenses/by/4.0/deed.de.

\section{Literatur}

Abrahamson, C.E. (1998). Storytelling as a pedagogical tool in higher education. Education, 118(3), 440-451.

Ainley, M., Hillmann, K., \& Hidi, S. (2002). Gender and interest processes in response to literary texts: situational inters and individual interest. Learning and Instruction, 12, 411-428.

Andrews, D. H., Hull, T.D., \& Donahue, J. A. (2009). Storytelling as an instructional method: descriptions and research questions. The interdisciplinary Journal of Problem-based Learning, 3(2), 6-23.

Arbuckle, J.L. (2015). IBM SPSS Amos 23 user's guide. New York, NY: IBM.

Asselborn, W., Jäckel, M., \& Risch, K. T. (2009). Chemie heute Sekundarstufe 1. Braunschweig: Westermann Schrödel.

Avraamidou, L., \& Osborne, J. (2009). The role of narrative in communication science. International Journal of Science Education, 13(12), 1683-1707.

Baltes-Götz, B. (2020). Mediator- und Moderatoranalysen mit SPSS und PROCESS. ZIMK Trier, https://www.uni-trier.de/fileadmin/ urt/doku/medmodreg/medmodreg.pdf. Zugegriffen: 2.11.2020.
Beerenwinkel, A., \& Gräsel, C. (2005). Texte im Chemieunterricht: Ergebnisse einer Befragung von Lehrkräften. Zeitschrift für Didaktik der Naturwissenschaften, 11, 21-39.

Berliner, D.C. (1992). Telling the stories of educational psychology. Educational Psychologist, 27(2), 143-161.

Browne, M., \& Cudeck, R. (1993). Alternative ways of assessing equation model fit. In K. A. Bollen \& J. S. Scott (Hrsg.), Testing structural equation models (S. 136-162). Newbury Park, CA: SAGE.

Byrne, B. M. (2001). Structural equation modelling with AMOS, basic concepts, applications and programming. Hillsdale, NJ: Lawrence Erlbaum.

Campbell, K. S. (1995). Coherence, continuity and cohesion: theoretical foundations for document design. Hillsdale, NJ: Erlbaum.

Chatman, S. (1978). Story and discourse. Narrative structure in fiction and film. Ithaca, NY: Cornell University Press.

Collins, F. (1999). The use of traditional storytelling in education to the learning of literacy skills. Early Child Development and Care, $152,77-108$.

Davies, J. (2010). Anthropomorphism in science. EMBO Reports, 11(10), 721

Dressler, W.U., \& Barbaresi, M. (1994). Morphogramatics. Diminutives and Intensifers in Italian, German and other languages. Berlin [u. a.]: Moutoun de Gruyter.

Duit, R., \& Glynn, S. (1992). Analogie und Metaphern, Brücken zum Verständnis im schülergerechten Physikunterricht. In P. Häussler (Hrsg.), Physikunterricht und Menschenbildung (S. 223-250). Kiel: IPN.

Egan, K. (1988). Teaching as Storytelling - an alternative approach to teaching and the curriculum. Padstow: T. J. Press.

Fechner, S. (2009). Effects of context-oriented learning on student interest and achievement in chemistry education. Berlin: Logos.

Fleischer, W., \& Barz, I. (2012). Wortbildung der deutschen Gegenwartssprache. Berlin [u. a.]: De Gruyter.

Flesch, R. (1951). How to test readability. New York: Harper.

Fludernik, M. (2006). Einführung in die Erzähltheorie. Darmstadt: Wiss. Buchges..

Folino, D. A. (2001). Stories and anecdotes in the chemistry classroom. Journal of Chemical Education, 78(12), 1615-1618.

Garner, R. (1992). Learning From School Texts. Educational Psychologist. 27(1), 53-63.

Genette, G. (2010). Die Erzählung. Paderborn: Fink.

Giessen, H.W. (2009). Emotion und Empathie. In H.W. Giessen (Hrsg.), Emotionale Intelligenz in der Schule: Unterrichten mit Geschichten (S. 7-18). Weinheim: Beltz.

Glynn, S. (2007). The teaching-with-analogies model. Science and Children, 44(8), 52-55.

Göttert, K.-H., \& Jungen, O. (2004). Einführung in die Stilistik. München: Fink.

Hair, J. F., Anderson, R. E., Tatham, R.L., \& Black, W.C. (1998). Multivariate data analysis. Upper Saddle River, NJ: Prentice-Hall.

Hayes, A. F. (2013). Introduction to Mediation, Moderation, and Conditional Process Analysis: A Regression-Based Approach. New York, NY: The Guilford Press.

Heering, P. (2013). Storytelling als Zugang zur Bildung in den Naturwissenschaften. In Früjahrstagung Didaktik der Physik. Jena. http://www.phydid.de/index.php/phydid-b/article/viewFile/443/ 582. Zugegriffen: 27. Juli 2019.

Heine, L., Domenech, M., Otto, L., Neumann, A., Krelle, M., Leiss, D., Höttecke, D., Ehmke, T., \& Schwippert, K. (2018). Modellierung sprachlicher Anforderungen in Testaufgaben verschiedener Unterrichtsfächer: Theoretische und empirische Grundlagen. Zeitschrift für angewandte Linguistik, 69, 69-96.

Heller, K., \& Perleth, C. (2000). KFT 4-12+R, Kognitiver FähigkeitenTest für 4. bis 12. Klassen: Revision-Materialien-Koffer. Göttingen: Beltz.

Hidi, S. (2006). Interest: a unique motivational variable. Educational Research Review, 1(2), 69-82. 
Hidi, S., \& Baird, W. (1988). Strategies for increasing text based-interest and students' recall of expository texts. Reading Research Quarterly, 23(4), 465-483.

Hidi, S., \& Berndorff, D. (1998). Situational interest and learning. In L. Hoffmann, A. Krapp, K. Renninger \& J. Baumert (Hrsg.), Interest and learning. Proceedings of the Seeon-conference on interest and gender (S. 74-90). Kiel: IPN.

Höft, L., Bernholt, S., Blankenburg, J.S., \& Winberg, M. (2019). Knowing more about things you care less about: cross-sectional analysis of the opposing trend and interplay between conceptual understanding and interest in secondary school chemistry. Journal of Research in Science Teaching, 56(2), 184-210.

Hulleman, C. S., Godes, O., Bryan, L. H., \& Harackiewicz, M. (2010). Enhancing interest and performance with a utility value intervention. Journal of Educational Psychology, 102(4), 880-895.

Jahrhaus, O. (2008). Grundkurs Literaturwissenschaft. Stuttgart: Klett.

Kalyuga, S., Chandler, P., \& Sweller, J. (1999). Managing split-attention and redundancy in multimedia instruction. Applied Cognitive Psychology, 13, 351-371.

Kaspar, L., \& Mikelskis, H.F. (2008). Lernen aus Dialogen und Geschichten im Physikunterricht - Ergebnisse einer Evaluationsstudie zum Thema Erdmagnetismus. Zeitschrift für Didaktik der Naturwissenschaften, 14, 7-25.

Kattmann, U. (2005). Lernen mit antropomorphen Vorstellungen? Ergebnisse von Untersuchungen zur Didaktischen Rekonstruktion in der Biologie. Zeitschrift für Didaktik der Naturwissenschaften, $11,165-174$.

Kline, R. B. (2005). Principles and practice of structural equation modelling. New York: Guilford.

Krapp, A. (1992). Das Interessenkonstrukt. Bestimmungsmerkmale der Interessenhandlung und des individuellen Interesses aus Sicht einer Personen-Gegenstands-Konzeption. In A. Krapp \& M. Prenzel (Hrsg.), Interesse, Lernen und Leistung. Münster: Aschendorff.

Krapp, A. (2002). An educational-psychological theory of interest and its relation to self-determination theory. In E.L. Deci \& R. M. Ryan (Hrsg.), Handbook of self-determination research (S. 405-427). Rochester: University of Rochester Press.

Kubli, F. (2001). Narrative Aspekte im naturwissenschaftlichen Unterricht. Zeitschrift für Didaktik der Naturwissenschaften, 7, 25-32.

Kubli, F. (2002). Plädoyer für Geschichten im Physikunterricht. Köln: Deubner.

Kubli, F. (2005). Mit Geschichten und Erzählungen motivieren. Köln: Deubner.

Langer, I., Schulz von Thun, F., \& Tausch, R. (2011). Sich verständlich ausdrücken. München: Reinhardt.

Lück, G. (2006). Geschichten erzählen im naturwissenschaftlichen Sachunterricht. Grundschule, 3, 43-45.

Martensen, M., Tietjens, K., \& Parchmann, I. (2007). Storytelling - eine Methode zur Kontextualisierung am Beispiel „Strom durch Chemie“. Mathematisch-naturwissenschaftlicher Unterricht. 60(7), 410-415.

Martin, L. (1999). Stories from the fields, hills, and labs. Journal of Museum Education, 24(3), 21-23.

Martiny, S.E., \& Fröhlich, L. (2020). Ein theoretischer und empirischer Überblick über die Entwicklung von Stereotypen und ihre Konsequenzen im Schulkontext. Wiesbaden: Springer.

Mayer, R.E. (2009). Multimedia learning. Cambridge [u.a.]: Cambridge University Press.

McQuiggan, S.W., Rowe, J.P., Mott, B.W., \& Lester, J.C. (2008). Story-based learning: the impact of narrative on learning experiences and outcomes. Lecture Notes in Computer Science, 5091, 530-539.

Miller, L. L. (1992). Molecular anthropomorphism. Journal of Chemical Education, 69(2), 141-142.

Ministerium für Schule und Weiterbildung des Landes NRW (2008). Kernlehrplan für das Gymnasium - Sekundarstufe I in NRW Chemie. Frechen: Ritterbach Verlag.
Norris, S.P., Guilbert, S. M., Smith, M.L., Hakimelahi, S., \& Phillips, L. M. (2005). A theoretical framework for narrative explanation in science. Science Education, 89(4), 535-563.

Paas, F.G.W.C. (1992). Training strategies for attaining transfer of problem-solving skill in statistics: a cognitive-load approach. Journal of Educational Psychology, 84(4), 429-343.

Püttschneider, M., \& Lück, G. (2004). Die Rolle des Animismus bei der Vermittlung chemischer Sachverhalte. CHEMKON, 11(4), 167-174.

Reschke, T. (2013). Entwicklung chemiebezogener Geschichten und die Evaluation ihrer Wirkung auf Fachwissen, situationales Interesse und Schülervorstellungen in der Sek I. Unveröffentlichte Masterarbeit Universität Osnabrück: Osnabrück.

Reschke (2017). Lesegeschichten im Chemieunterricht der Sekundarstufe I zur Unterstützung von situationalem Interesse und Lernerfolg. Berlin: Logos.

Rheinberg, F., Vollmeyer, R., \& Burns, B.D. (2001). FAM: Ein Fragebogen zur Erfassung aktueller Motivation in Lern- und Leistungssituationen. Potsdam [u. a.]. http://www.psych.unipotsdam. de/people/rheinberg/messverfahren/FAMLangfassung.pdf. Zugegriffen: 8. Sept. 2016.

Rotgans, J.I., \& Schmidt, H. G. (2014). Situational interest and learning: thirst for knowledge. British Educational Research Journal, $43,350-371$.

Rotgans, J. I., \& Schmidt, H. G. (2017). The relation between individual interest and knowledge acquisition. Learning and Instruction, 32(2), 37-50.

Schaffner, E. (2009). Effekte kognitiver und motivationaler Faktoren auf das Verstehen und Lernen von Texten. Berlin. http:// edocs.fuberlin.de/diss/servlets/MCRFileNodeServlet/FUDISS derivate_000000006738/Schaffner_Effekte_habitueller_Lesemo tivation_II.pdf?hosts=. Zugegriffen: 1. Juli 2016.

Schiefele, U. (1996). Motivation und Lernen mit Texten. Göttingen: Hogrefe.

Schiefele, U., \& Krapp, A. (1996). Topic interest and free recall of expository text. Learning and Individual Differences. 8(2), 141-160.

Schmidt, H. G., \& Rotgans, J.I. (2017). Like it or not: individual interest is not a cause but a consequence of learning. Rejoinder to Hidi and Renninger (2017). British Educational Research Journal, 43(6), 1266-1268.

Schneider, W., Schlagmüller, M., \& Ennemoser, M. (2007). Lesegeschwindigkeits- und -verständnistest für die Klassen 6-12 $(L G V T)$. Göttingen: Hogrefe.

Schraw, G. (1997). Situational interest in literary text. Contemporary Educational Psychology, 22(4), 436-456.

Schraw, G., \& Lehman, S. (2001). Situational Interest: A review of the Literature and Directions for Future Research. Educational Psychology Review. 13(1), 23-52.

Stanat, P., Schipolowski, S., Mahler, N., Weirich, S., \& Henschel, S. (2018). IQB-Bildungstrend 2018. Münster: Waxmann. https:// www.iqb.hu-berlin.de/bt/BT2018/Bericht [30.12.2019]

Stanzel, F. K. (1998). Theorie des Erzählens. Göttingen: Vandenhoeck \& Ruprecht.

Sweller, J., Ayres, P., \& Kalyuga, S. (2011). Cognitive Load Theory. Explorations in the learning sciences, instructional systems and perfomance technologies. New York [u. a.]: Springer.

Talanquer, V. (2013). When atoms want. Journal of Chemical Education, 90(11), 1419-1424.

Tapola, A., Veermans, M., \& Niemivirta, M. (2013). Predictors and outcomes of situational interest during a science learning task. Instructional Science, 41(6), 1047-1064.

Toolan, M. J. (2001). Narrative: a critical linguistic introduction. London: Routledge.

Urban, D., \& Mayerl, J. (2011). Regressionsanalyse: Theorie, Technik und Anwendung. Wiesbaden: Springer VS.

Wellington, J., \& Osborne, J. (2001). Language and literacy in science education. Buckingham, UK: Open University Press. 